\title{
Setting tolerance limits for statistical project control using earned value management
}

\author{
Jeroen Colin ${ }^{\mathrm{a}}$, Mario Vanhoucke $\mathrm{a}^{\mathrm{a}, \mathrm{b}, \mathrm{c}, *}$ \\ ${ }^{a}$ Faculty of Economics and Business Administration, Ghent University, Tweekerkenstraat 2, 9000 Gent \\ (Belgium) \\ ${ }^{b}$ Technology and Operations Management, Vlerick Business School, Reep 1, 9000 Gent (Belgium) \\ ${ }^{c}$ Department of Management Science and Innovation, University College London, Gower Street, London WC1E \\ 6BT (United Kingdom)
}

\begin{abstract}
Project control has been a research topic since decades that attracts both academics and practitioners. Project control systems indicate the direction of change in preliminary planning variables compared with actual performance. In case their current project performance deviates from the planned performance, a warning is indicated by the system in order to take corrective actions.

Earned value management/earned schedule (EVM/ES) systems have played a central role in project control, and provide straightforward key performance metrics that measure the deviations between planned and actual performance in terms of time and cost. In this paper, a new statistical project control procedure sets tolerance limits to improve the discriminative power between progress situations that are either statistically likely or less likely to occur under the project baseline schedule. In this research, the tolerance limits are derived from subjective estimates for the activity durations of the project. Using the existing and commonly known EVM/ES metrics, the resulting project control charts will have an improved ability to trigger actions when variation in a project's progress exceeds certain predefined thresholds

A computational experiment has been set up to test the ability of these statistical project control charts to discriminate between variation that is either acceptable or unacceptable in the duration of the individual activities. The computational experiments compare the use of statistical tolerance limits with traditional earned value management thresholds and validate their power to report warning signals when projects tend to deviate significantly from the baseline schedule.
\end{abstract}

Keywords: Project Management, Scheduling, Risk, Simulation

\section{Introduction}

Project management and control have been research topics since the development of project planning approaches such as the critical path method (CPM, [1, 2]) and the Program Evaluation and Review Technique (PERT, [3]). The majority of the research endeavors published in the academic literature focuses on the construction of a project baseline schedule within the presence of limited resource constraints (see e.g. a recent survey written by Hartmann and Briskorn [4]). Ever since, the use of a project baseline schedule has been put into the right perspective, as it only acts as a

\footnotetext{
${ }^{*}$ Corresponding author

Email addresses: jeroen.colin@ugent.be (Jeroen Colin), mario.vanhoucke@ugent.be (Mario Vanhoucke)
} 
roadmap for the future project performance. If the project runs into trouble, the baseline schedule can be used to detect such problems and to trigger corrective actions. The construction of a project baseline schedule should go hand in hand with the development of a risk analysis [5] to detect the weak parts in the schedule. These two sources of information should therefore be used concurrently, both before and during the execution of the project. The need for the integration of the project baseline scheduling construction, the risk analysis and management and the project control step has been recently referred to as dynamic scheduling $[6,7]$.

In this paper, the construction of a baseline schedule is assumed to be a given, and the focus lies on the presentation of a new project control system [8]. Rozenes et al. [9] have characterized a project control system by stating that it should indicate the direction of change in preliminary planning variables when compared with actual performance. If there is an unacceptable gap between planning and performance, a warning should be generated by the system in order to take corrective action. In the remainder of this text, we will further elaborate on this unacceptable gap and quantify how it can be specified.

This study mainly focuses on the generation and interpretation of warning signals during project control and hence it does not include results in the quality and input of corrective actions, nor on the use of these new methods for contingency planning.

A well-known and widely used project control system is Earned Value Management (EVM) originally developed in the '60s at the U.S. Department of Defense as a standard method to measure a project's performance. This system relies on a set of straightforward metrics to measure and evaluate the general health of a project. These metrics serve as early warning signals to timely detect project problems or to exploit project opportunities. EVM has been investigated and used widely in project management practice and has been brought into the attention of researchers by Fleming and Koppelman [10]. Recently, the research on EVM has produced the earned schedule method (ES, [11]) and a large study on the integration of risk management and EVM/ES was conducted [12].

EVM/ES calculations are based on the costs and durations for the activities in a project. Both in the planning phase and during the execution of the project, some detail of the individual activities is lost as the individual performance is summed up into the performance metrics observed and controlled at the project level. Earned value management is known to have some shortcomings when it comes to protecting the critical path in the schedule [10]. The summation of activity earned value into the aggregate level for the project does not incorporate schedule risk analysis (SRA, [13]) data, such as criticality. In spite of the complexity of this problem, EVM/ES use in practice is characterized by decision making from practical experience, rules-of-thumb and anecdotal evidence. In the academic literature, it is reasoned that the application of a statistical process control approach could benefit the EVM/ES project control process. These previous attempts depend on the availability of historical records that may or may not exist and may or may not be valid. We therefore also allow the use of subjective estimates.

In this paper, a new statistical project control approach is presented. It is inspired by the practical format of statistical process control charts and based on tolerance intervals produced by a simulated sample. A controlled simulation experiment will be used to specifically produce two types of project progress situations. First, project progress situations where the variation from plan is limited to some acceptable margin are used to produce project control charts. Secondly, we test the discriminative power of these control charts when the variation from plan exceed the predefined acceptable margin.

The outline of this paper is as follows. In section 2, we present a literature review on the use of statistical control principles in project management and control. We will present two project control charts, the X chart and R chart. Section 3 illustrates these control charts on a fictitious project example. In section 4 , the design of the computational test experiments is outlined based on various sources from literature. In section 5 , test results obtained by computational experiments 
using Monte Carlo simulation to imitate a fictitious project progress environment are discussed. Section 6 draws general conclusions and highlights future research avenues.

\section{Statistical Project Control}

\subsection{Literature overview}

This literature review introduces the main concepts of statistical process control (SPC) as they are applied to monitor and control processes in services and manufacturing. The application of $\mathrm{SPC}$ techniques to earned value management is not new in literature. We introduce a statistical project control procedure that aims to overcome some of the issues with the previously published approaches, but which is not an implementation of SPC in the strict sense, although it reproduces some of its concepts and nomenclature. The last paragraph of this literature review is dedicated to a summation of the differences between our statistical project control procedure and the implications of applying SPC to project control in a strict sense.

In standard SPC applications a state of control is identified with a process generating samples for which the subgroup averages are approximately normal under the central limit theory. Control charts such as the Shewhart, cumulative sum (CUSUM) and exponentially weighted moving avarage (EWMA) charts serve as on-line procedures to monitor process stability, to detect assignable variation or to forecast process movements in industrial processes [14]. A process is said to be in-control when only common cause variation is present. This type of variation is characterized as coming from phenomena constantly active in a process, which can be predicted probabilistically. In his original work on process control, Shewhart [15] introduced the term chance cause. A process is said to be out-of-control if a second type of variation is present known as assignable cause variation. Assignable cause variation arises when a new, previously unanticipated phenomenon is present in the system and should cause a signal.

Figure 1 illustrates how a control chart applied to project control might be interpreted. Periodic observations of a performance measure for a fictitious project execution are outlined, along with an illustrative upper control limit (UCL) and lower control limit (LCL). For illustrative purposes, we will assume that the performance measure is of the type where a high value is good and a low value acts as a trigger for actions due to potential problems. The frequently encountered schedule performance index (SPI, section 2.3.1) and schedule performance index using earned schedule (SPI(t), section 2.3.1) measures in EVM/ES are of this type, although control charts of the opposite type have also been proposed where the natural logarithm of the reciprocal of SPI or $\mathrm{SPI}(\mathrm{t})$ is taken [16]. In case the observations fall outside the project control limits, the charts report a signal which could trigger actions to bring the project back to plan or to exploit opportunities. More precisely, when an observation falls below the lower control limit, the performance has likely dropped below the pre-defined acceptable margin on the baseline estimates. In this case, this signal should be interpreted as a warning signal to the project manager to consider taking corrective actions. When the performance indicator exceeds the upper control limit, it can be seen as a signal to exploit opportunities, in which case the current project schedule might be re-baselined to incorporate this opportunity. Different SPC control charts have been implemented for project control using earned value management by [16-21]. A short overview of their respective approaches and findings is listed below.

Lipke and Vaughn [17] apply a XmR chart (individuals and moving range chart) and calculate the control limits based on a two-observation sample of earned value performance metrics. They use the reciprocal measures of CPI (cost performance index) and SPI (schedule performance index), as they have shown that these are normalized, such that many of the issues of applying SPC, such as variability and homogeneity of the data, are avoided. Bauch and Chung [18] modify the statistical process control charts proposed by Shewhart to be used with earned value management considering three major aspects. Firstly, they asses single observations relative to historical project 


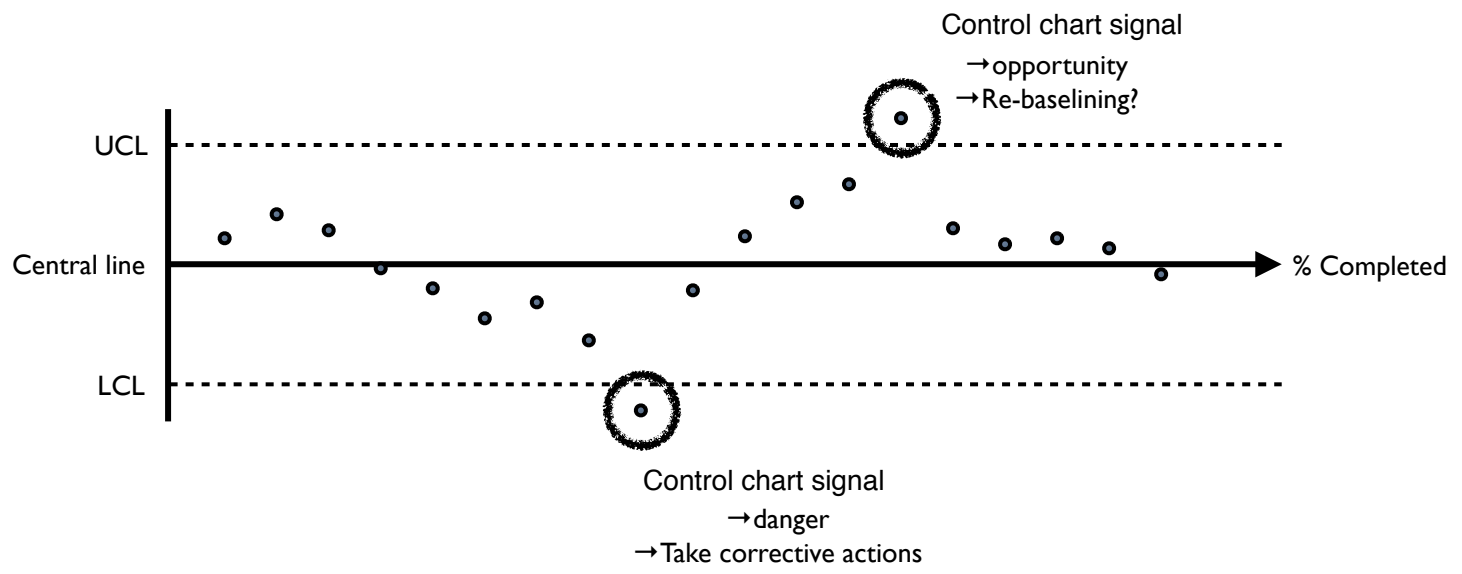

Figure 1: Illustrative control chart and out-of-control indications

data from 20 similar projects. Secondly, as projects span a finite time horizon, which is typically different for each project, they normalize the different time lengths of each project into a consistent number of time periods, chosen as 20 discrete measurements for the purpose of the paper. As a third modification, they adopted the CUSUM approach [22] to incorporate the increasing nature of period-to-period measurements of project performance. The typical y-axis of control charts is transformed to plot cumulative project performance values. As a result, the central line and upper and lower limits are non-stationary. Wang et al. [19] illustrate the use of SPC charts using EVM/ES on a set of more than 30 software projects where abnormal progress situations are effectively detected. [20] combine XmR charts with EVM/ES performance metrics, but implement the logarithm of the reciprocal of SPI and the cost performance index (CPI). This was earlier proposed by Lipke [16]. Control limits are calculated from historical data of 120 projects performed by a management consulting company. Leu and Lin [20] introduce the division of different cases based on historical factors influencing project performance and quantify the XmR chart procedure for different trends observed in their data. Moreover, they accurately describe how "the quick proliferation and complexity of project performance data indicate the need for a well-organized project performance analysis process." Aliverdi et al. [21] suggest to transform CPI data when quality control charts are applied for cost control. SPC charts for both individual and moving range measurements were successfully implemented. They conclude that the integrated approach of EVM/ES and statistical quality control charts can contribute to a better and more reliable project control process.

Overall, previous research on the integration of EVM/ES with SPC techniques for projects has established that it improves project control by providing an objectively based and easily implemented real-time monitoring system. The evidence provided in the literature is based on empirical research of either post-hoc statistical analyses on projects [19] or the implementation of control limits derived from historical data $[16,20]$. The dependency on historical data does not pose an immediate problem for statistical project control approaches, ideally the use of historical records is preferred over subjective estimates for project control [23]. However, the concept of "similarity" between projects, is often vaguely defined. When historical EVM/ES data is directly applied, the project-specific EVM/ES dynamics should not be ignored. [24] illustrated these project-specific dynamics with EVM/ES forecasting becoming less reliable for projects with a network close to a parallel network structure. When the concepts of SPC are directly translated to incorporate EVM/ES measurement, an assumption with respect to their distribution has to be made. In previous literature this prerequisite was fulfilled by transforming the data to appear normally distributed $[17,19]$ or lognormally distributed [16, 20] or appeared overlooked [21].

In section 2.3 we propose a different statistical project control procedure that will also include 
the appealing aspects of using control charts. They are easy to set up, implement and interpret. However, the imposed tolerance limits on the earned value metrics should be interpreted in the more technical sense [25]. The tolerance limits are acquired from the empirical distribution function (edf) of the EVM/ES metric under study at different points along the life-cycle of a project. This edf is obtained from a Monte Carlo simulation model that allows variation to be added at the activity level of the project. This variation can be introduced as subjective estimates of what deviations are statistically plausible during the execution of the project or from calibration with historical in-company data.

Table 1: Difference between SPC for projects and our statistical project control procedure

\begin{tabular}{|c|c|c|}
\hline & $\begin{array}{l}\text { statistical process control } \\
\text { for project control }\end{array}$ & $\begin{array}{l}\text { statistical project control } \\
\text { setting tolerance limits }\end{array}$ \\
\hline Data type & Process data & Simulated data \\
\hline Data measurements & Data collected over time & Data collected using simulations \\
\hline Number of data points & Small sample size per time unit & Large sample size per simulation point \\
\hline Process & Infinite process & Finite process \\
\hline Analysis & Ex post & Ex ante \\
\hline Control limits & Deviations from normal progress & Deviations from desired progress \\
\hline Reference value & $\begin{array}{l}\text { Normal project progress } \\
\text { (with normal variation) }\end{array}$ & $\begin{array}{c}\text { Baseline schedule } \\
\text { (with acceptable tolerances) }\end{array}$ \\
\hline
\end{tabular}

Table 1 summarizes the main differences between our statistical project control procedure that uses tolerance limits and a classical SPC implementation for project control. The main aspects are given along the following lines.

- Data input: A first fundamental difference lies in the data input necessary to construct the control limits. While process data needs to be collected at regular time intervals to monitor the behavior of a process in progress when classical SPC is implemented, our control charts rely on inputs of estimates that express the desired tolerance on the activity durations or allows calibration to historical data at the activity level. Consequently, these inputs are used to express an ex ante desired state of outcome on activity durations rather than an observed ex post outcome in the measured variable. For this reason, the activity distribution inputs can and probably will be different than the real activity distributions, and the tolerance limits are set up to detect these differences and to receive timely warnings when real activity durations exceed the desired thresholds. Unlike classical applications of SPC to project control, which aim at detecting deviations from the average on-going state of the measurable variable in progress, the project control charts using tolerance limits aim at detecting deviations from a predefined desired state in the variable defined before the start of the progress.

- Dynamic project performance measurements: Given the ex ante approach of setting tolerance for project control, the procedure requires a large sample of data points to predict the impact of the acceptable activity tolerances on the tolerance limits set for EVM/ES. Therefore, the data is collected using Monte-Carlo simulations using the desired tolerances on the activity distributions and measuring their impact on the EVM/ES output metrics such as SV, SPI, $\mathrm{SV}(\mathrm{t})$ and $\mathrm{SPI}(\mathrm{t})$. Consequently, these output metrics are used to construct tolerance limits given the desired input parameters. This is in high contrast to the on-going data collection method of observed process data used to construct control limits to detect deviations from normal or average process behavior in a classical application of SPC.

- Control limits: Due to the fundamental difference between the control limits of SPC for project control and the statistical tolerance limits based on the desired activity duration tolerances of our project control approach, we have decided to use the words tolerance limit 
rather than control limit during the construction of the control charts. Consequently, the control limits set on the EVM/ES output metrics SV, SPI, SV(t) and SPI(t) are used to define the thresholds on the tolerances in project performance behavior given the desired tolerances on the activity durations and hence act as warning signals to measure unacceptable project progress behavior compared to the baseline schedule. This is exactly what an EVM/ES system is trying to accomplish and this view is consistent with the definition of a project control system [9]. Project based performance metrics along the project progress are used to act as an action threshold to detect underlying problems that are responsible for the deviation between planned outcome and project progress outcome. The tolerance limits used in this paper define these action thresholds based on a statistical analysis of the simulated data resulting from a pre-specified allowable variation.

\subsection{A new statistical project control approach}

Our statistical project control approach differs from those described in the literature overview by setting tolerance limits. These tolerance limits are calculated by means of simulation and this allows us to redefine and adapt the process control principles regularly found in manufacturing and services to our requirements. Process control is generally an ongoing process where little in-depth information is known for the process, and a state of statistical control is inferred from the characteristics of the recorded process variables. Our approach, shares the use of control charts, but is fundamentally and functionally different, as outlined in the last paragraph of section 2.1 .

Practically, these differences brings about two main aspects:

- The statistical control procedure for a project will be finite in time. This follows directly from the definition of a project as a one-time endeavour with a clearly defined start and finish. The control chart will therefore only allow a predefined number of measurements at distinct time-instances.

- Simulation allows us to apply a state of control, such as the user deems appropriate, and characterize the resulting EVM/ES project progress variables. Historical data can play a role in the input modelling of the simulation, but is no longer directly required to calculate the control limits. Historical records of EVM/ES may be used to evaluate the congruence of the simulation output with real-life executions.

The main research interest of this article is to develop and test a new project control approach for schedule control. Since schedule control can be expressed in the deviations between the actual durations and the planned durations of the activities of the project, these will be the primary focus of our simulation model. The simulation model will add uncertainty to the durations of the activities, which will result in fictitious project executions that deviate from the baseline. An effective statistical project control chart is required to differentiate between the variation that is acceptable for an activity and variation that is unacceptable. If an activity has a deviation between its real duration and its planned durations that is unacceptably large, we wish to detect this on a project control chart using EVM/ES. If all the activity durations lie within pre-defined acceptable limits, we will consider the project to be executed as planned, and the control charts should not generate any signal.

Our approach allows the user to set tolerances on the activity level, which will result in tolerance limits for project progress variables to be used at the project level in control charts. The tolerance limits for the project progress variables will be calculated in what is referred to as the static phase of our procedure.

When actual project progress is recorded during the execution of a project, the observations can be plotted against the tolerance limits on the project control charts. This phase is referred to as the dynamic phase. In section 5, we will quantify the performance of the tolerance limits in the control charts using a second large Monte Carlo simulation. 


\subsection{Project control charts}

\subsubsection{The static phase}

Determine the as planned situation. If the project in progress exhibits an abnormal behavior compared to a predefined situation, the project manager must be able to timely detect this. Consequently, the normal project progress situation needs to be defined by the project manager in order to construct control charts that distinguish between as planned and not as planned situations. Therefore, it is assumed that the project baseline schedule is a forecast of what might happen in the future during project progress and determines the as planned situation, subject to some acceptable variation on the activity durations and possible project-wide effects of uncertain events. This is exactly the starting point of dynamic scheduling, which proposes that the project baseline schedule needs to be used to measure the project progress. Both positive and negative deviations within a certain range are assumed to be inherent to any project and are considered as normal while abnormal deviations exceeding certain thresholds should trigger further investigations.

This variation on the activity durations, and additional uncertain events, obviously have an impact on the project performance which might differ from the initially expected project performance, as stipulated in the project baseline schedule. This as planned project performance deviation will be measured by Monte Carlo simulation runs.

Monte Carlo simulations. Monte Carlo simulations in project management and control have been used widely in literature. Since its introduction [26] to analyze project networks, the methodology has been used for a range of project management research projects and applications. In [27], a classified bibliography of project risk management research is given where simulation plays a central role. Ever since, Monte Carlo simulation studies have been a respected methodology in project management and scheduling. The simulation studies in this paper are based on the project control simulation studies described in $[28,29]$. The methodology used has been initially developed by Vanhoucke and Vandevoorde [24] and is formalized in a dynamic scheduling simulation tool, P2 Engine [30].

In the current paper, the simulation runs will be used to simulate fictitious project progress in order to obtain periodic project performance data using the well-known project performance indicators obtained by the EVM/ES key metrics. Consequently, fictitious project progress is simulated for a number nrs of Monte Carlo runs. Variation in the activity durations will have an impact on the project performance over time and therefore, the variable $X_{s p}$ will be used to denote the performance indicator value from simulation run $s(s=1, \ldots, n r s)$ measured at a certain moment in time between the start and the finish of the simulated project progress when it is $p$ percent completed $(p=(1, \ldots, P) \times \Delta \mathrm{PC})$. A total of $P$ observations are taken with increment $\Delta \mathrm{PC}$.

The following performance indicators are periodically measured during the simulation runs

$\mathrm{SV} \quad$ Schedule variance $(\mathrm{SV}=\mathrm{EV}-\mathrm{PV})$

SPI $\quad$ Schedule performance index $(\mathrm{SPI}=\mathrm{EV} / \mathrm{PV})$

$\mathrm{SV}(\mathrm{t}) \quad$ Schedule variance using earned schedule $(\mathrm{SV}(\mathrm{t})=\mathrm{ES}-\mathrm{AD})$

$\mathrm{SPI}(\mathrm{t}) \quad$ Schedule performance index using earned schedule $(\mathrm{SPI}(\mathrm{t})=\mathrm{ES} / \mathrm{AD})$

with

$\begin{array}{ll}\text { PV } & \text { Planned value } \\ \text { EV } & \text { Earned value } \\ \text { AC } & \text { Actual cost } \\ \text { ES } & \text { Earned schedule } \\ \text { AD } & \text { Actual duration } \\ \text { BAC } & \text { Budget at completion } \\ \text { PC } & \text { Percentage project completion }(\mathrm{PC}=\mathrm{EV} / \mathrm{BAC})(0 \% \leq \mathrm{PC} \leq 100 \%)\end{array}$ 
For the sake of completeness, we even include SV and SV(t) in our research. Previous EVM/ES studies using SPC often neglected these performance metrics and thus very few empirical evidence for their distributions is known. Since we do not assume any distribution for the control measures a priori, we can incorporate them into our research. Note that, we do not incorporate the cost control metrics cost variance $(\mathrm{CV}=\mathrm{EV}-\mathrm{AC})$ and cost performance index ( $\mathrm{CPI}=\mathrm{EV} / \mathrm{AC})$. In our simulation model, cost is always strongly correlated with the time-performance of the activity, which might not necessarily be the case in real-life. Since our focus is on controlling the time performance of a project, we restrict the analysis to those metrics specifically designed to do so.

Add tolerance limits. The simulated data is used to construct control limits for the project control variables $X_{p}=(\mathrm{SV}, \mathrm{SV}(\mathrm{t}), \mathrm{SPI}, \mathrm{SPI}(\mathrm{t}))$ at a review period $p$ for two distinct types of control charts. The proposed $\mathrm{X}$ chart and the $\mathrm{R}$ chart resemble the Shewhart individuals (indX) and moving range $(\mathrm{mR})$ control charts in their ability to inspect individual observations [31]. Control limits for the Shewhart charts are constructed based on the moving average over the range (i.e. the difference between an observation $x_{i}$ and its predecessor $x_{i-1}$ ), calculated using the previous $m-1$ observations.:

$$
\widehat{\mathrm{MR}}=\frac{1}{m-1} \sum_{i=2}^{m}\left|x_{i}-x_{i-1}\right|
$$

This concept of moving range is not adopted here to construct tolerance limits for project control, since it implies that observations are taken for a consistently operating process. On the contrary, we assume that our nrs simulation runs provide sufficient observations to reconstruct the distribution function for the variable $X_{p}$ at a review period $p \in(1, \ldots, P) \times \Delta \mathrm{PC}$. Note that we do not assume two random variables $X_{j}$ and $X_{k}$ (where $j, k \in(1, \ldots, P) \times \Delta \mathrm{PC}$ ) to be independent, but we will construct tolerance limits for each of them independently. For any two adjacent review periods, we will look at the range $R_{j}=\left|X_{j}-X_{j-1}\right|(j \in(1, \ldots, P) \times \Delta \mathrm{PC})$ and treat this variable as a new project performance variable.

- The X chart is used to monitor individual observations for the project performance variables $X_{p}$ at a review period $p$. Tolerance limits can be calculated from the empirical distribution function $\hat{\mathrm{F}}_{X_{p}}(t)$.

$$
\hat{\mathrm{F}}_{X_{p}}(t)=\frac{1}{n r s} \sum_{s=1}^{n r s} \mathbb{1}\left\{X_{s p} \leq t\right\}
$$

derived from a sample of independent identically distributed (i.i.d.) variables $\left(X_{1 p}, \ldots\right.$, $\left.X_{n r s p}\right)$ taken at $p . \mathbb{1}\{A\}$ represents the indicator function of an event $A$ :

$$
\mathbb{1}\{A\}= \begin{cases}1 & \text { if } A \text { is TRUE } \\ 0 & \text { if } A \text { is FALSE }\end{cases}
$$

By the strong law of large numbers, we know that the emperical distribution function (2) asymptotically converges to the real cumulative distribution function $\mathrm{F}(t)$ for all possible values of $t$.

The lower and upper statistical tolerance limits at a review period $p$ for a level $\alpha\left(L T L_{X_{p}}^{\alpha}\right.$ and $\left.U T L_{X_{p}}^{\alpha}\right)$ are then calculated as the $\alpha^{\text {th }}$ and $(1-\alpha)^{\text {th }}$ quantile of the distribution $\mathrm{F}(t)$. The quantiles can be calculated from the inverse of the empirical distribution function or by estimation of the sample quantiles. Let $\hat{Q}(\alpha)_{p}$ for $0<\alpha<1$ denote the sample quantile based on a a set of independent observations $\left\{X_{1 p}, X_{2 p}, \ldots, X_{n r s p}\right\}$ from the distribution $F$ at review period $p$. If the order statistics of $\left\{X_{1 p}, X_{2 p}, \ldots, X_{n r s p}\right\}$ are denoted as 
$\left\{X_{(1) p}, X_{(2) p}, \ldots, X_{(n r s) p}\right\}$, then $\hat{Q}(\alpha)_{p}$ can be estimated as:

$$
\begin{aligned}
\hat{Q}(\alpha)_{p}= & (1-\gamma) X_{(j) p}+\gamma X_{(j+1) p} \\
& \text { where } \frac{j-\alpha+1}{n r s} \leq \alpha<\frac{j-2+\alpha}{n r s}
\end{aligned}
$$

with $\gamma=\alpha$ nrs $-\alpha-j+1$ and $j=\lfloor\alpha n r s-\alpha+1\rfloor$. $\lfloor u\rfloor$ is used to represent the largest integer smaller than $u$. We refer to [32] for a detailed comparison of different functions to calculate continuous sample quantiles.

The lower and upper statistical tolerance limits at a review period $p$ for a level $\alpha$ for the $\mathrm{X}$ chart are:

$$
\begin{aligned}
L T L_{X_{p}}^{\alpha} & =\hat{Q}(\alpha)_{p} \\
U T L_{X_{p}}^{\alpha} & =\hat{Q}(1-\alpha)_{p}
\end{aligned} \quad \forall p \in(1, \ldots, P) \times \Delta \mathrm{PC}
$$

The value for the level $\alpha$ can be chosen subsequently with respect to a total level of control over al $P$ review periods of the project. We will illustrate this in section 5.1.

- The $\mathrm{R}$ chart monitors the range $R$ (or difference) between two adjacent observations. This results in tolerance limits for a vector of observations which is in length one less than those for the $\mathrm{X}$ chart. The empirical distribution function $\hat{\mathrm{F}}_{R_{p}}(t)$ for $R$ at a period $p$ can be found using nrs simulation runs.

$$
\hat{\mathrm{F}}_{R_{p}}(t)=\frac{1}{n r s} \sum_{s=1}^{n r s} \mathbb{1}\left\{R_{s p} \leq t\right\}
$$

with:

$$
R_{s p}=\left|X_{s p}-X_{s p-1}\right| \quad \forall p \in(2, \ldots, P) \times \Delta \mathrm{PC}
$$

and the tolerance limits for a level $\alpha$ are:

$$
\begin{aligned}
L T L_{R_{p}}^{\alpha} & =\hat{Q}(\alpha)_{p} \\
U T L_{R_{p}}^{\alpha} & =\hat{Q}(1-\alpha)_{p}
\end{aligned} \quad \forall p \in(2, \ldots, P) \times \Delta \mathrm{PC}
$$

where $\hat{Q}(\alpha)_{p}$ is calculated with the order statistics $\left\{R_{(1) p}, R_{(2) p}, \ldots, R_{(n r s) p}\right\}$. The intuitive explanation for the use of the range chart is that the difference between two measurements of project performance relates to the instantaneous change of the performance. Since a project consists of activities that begin and end at discrete time instances, the transformed variable $R_{p}$ should better reflect the performance of activities that are being executed at that time instance and the project control process is less cluttered by the performance of past activities.

\subsubsection{The dynamic phase}

A project needs to be monitored by periodically measuring its performance. Each periodic project performance observation can be plotted on the control chart. $Y_{p}$ is used to refer to the performance indicator value during project progress at an instance $p$. It is different from $X_{s p}$ in the sense that it is not a priori assumed to be drawn from the distribution of $X_{s p}$. Rather, the calculated tolerance limits for the control charts are used to asses whether a value $Y_{p}$ is likely to have been produced under the null hypothesis (characterized by the empirical distribution function of $X_{p}$ in the simulation of the static phase).

Classical SPC applications often reject the notion that control charts are a form of hypothesis testing. However, using tolerance limits for our statistical project control approach, we have taken 
the liberty to present our control charts in such a way. Control charts and hypothesis tests can be regarded as alternatives and the analogy is as presented below:

The null and alternative hypotheses of the project schedule control procedure proposed in this paper are

$$
\begin{aligned}
& \mathrm{H}_{0} \text { : The project is executed as planned } \\
& \mathrm{H}_{\mathrm{a}} \text { : The project is executed not as planned }
\end{aligned}
$$

This hypothesis is tested using periodic control charts, by checking whether the current project performance $Y_{p}$ lies within the control limits or not, as follows

$$
\begin{aligned}
\text { Accept } \mathrm{H}_{0}: L T L_{X_{p}}^{\alpha} \leq Y_{p} \leq U T L_{X_{p}}^{\alpha} \\
\text { Reject } \mathrm{H}_{0}:\left(Y_{p}<L T L_{X_{p}}^{\alpha}\right) \vee\left(Y_{p}>U T L_{X_{p}}^{\alpha}\right)
\end{aligned}
$$

for the X chart and

$$
\begin{aligned}
& \text { Accept } \mathrm{H}_{0}: L T L_{R_{p}}^{\alpha} \leq\left|Y_{p}-Y_{p-1}\right| \leq U T L_{R_{p}}^{\alpha} \\
& \text { Reject } \mathrm{H}_{0}:\left(\left|Y_{p}-Y_{p-1}\right|<L T L_{R_{p}}^{\alpha}\right) \vee\left(\left|Y_{p}-Y_{p-1}\right|>U T L_{R_{p}}^{\alpha}\right)
\end{aligned}
$$

for the $\mathrm{R}$ chart.

\section{Illustrative example}

This section illustraties the calculations to construct statistical project control charts based on a fictitious project network example. Figure 2 displays the fictitious illustrative project network from [12] with 10 non-dummy activities. The numbers above each node are used to display the predefined baseline duration (in days) while the number below each node denotes the baseline total activity cost.

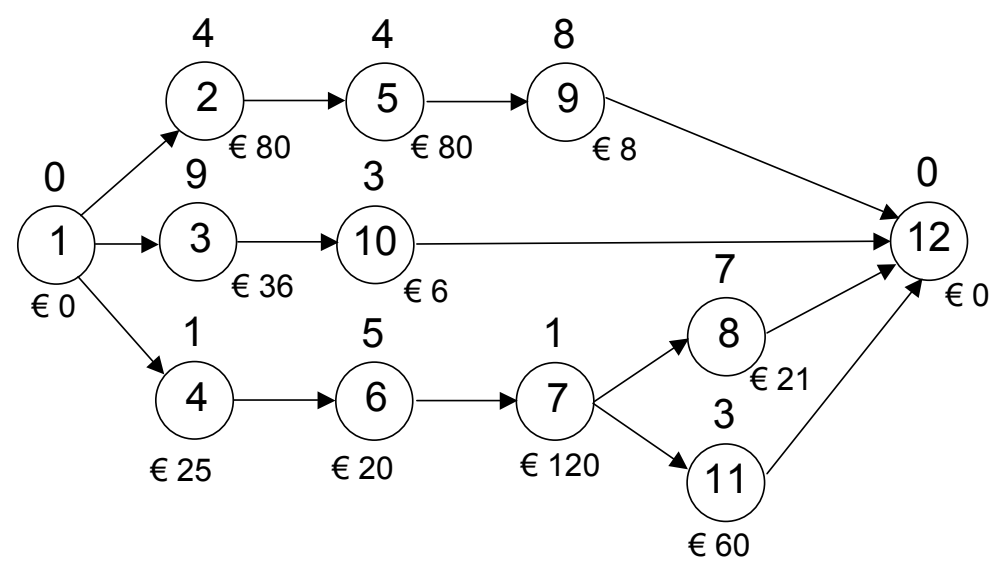

Figure 2: An illustrative project network (Source: [12])

Table 2 displays five simulation runs ( $n r s=5)$ where the activity durations have been randomly chosen from a uniform distribution with a maximum relative deviation from the baseline duration equal to $30 \%$. The first row of table 2 shows the limits between which the fictitious activity durations can be chosen.

Table 3 displays the earned value metric EV along the project duration (in days). It is assumed that all activities follow a linear earned value accrue and, consequently, variation in an activity duration has a linear impact on the actual activity cost. 
Table 2: Normal activity variation (in days) from five simulation runs

\begin{tabular}{cccccccccccc}
\hline Activity & $\mathbf{2}$ & $\mathbf{3}$ & $\mathbf{4}$ & $\mathbf{5}$ & $\mathbf{6}$ & $\mathbf{7}$ & $\mathbf{8}$ & $\mathbf{9}$ & $\mathbf{1 0}$ & $\mathbf{1 1}$ & $\mathbf{R D}$ \\
\hline$c \pm \delta^{c v}$ & $3-5$ & $6-12$ & $1-1$ & $3-5$ & $4-6$ & $1-1$ & $5-9$ & $6-10$ & $2-4$ & $2-4$ & \\
\hline $\mathbf{1}$ & 3 & 8 & 1 & 5 & 4 & 1 & 9 & 6 & 2 & 4 & 15 \\
$\mathbf{2}$ & 5 & 6 & 1 & 5 & 6 & 1 & 7 & 6 & 3 & 3 & 16 \\
$\mathbf{3}$ & 4 & 7 & 1 & 4 & 5 & 1 & 6 & 7 & 2 & 3 & 15 \\
$\mathbf{4}$ & 4 & 12 & 1 & 3 & 5 & 1 & 9 & 8 & 4 & 2 & 16 \\
$\mathbf{5}$ & 3 & 11 & 1 & 3 & 4 & 1 & 8 & 10 & 2 & 4 & 16 \\
\hline \hline
\end{tabular}

Table 3: The Earned Value (EV) and Planned Value (PV) along the project duration

\begin{tabular}{|c|c|c|c|c|c|c|c|c|c|c|c|c|c|c|c|c|}
\hline Scenario & 1 & 2 & 3 & 4 & 5 & 6 & 7 & 8 & 9 & 10 & 11 & 12 & 13 & 14 & 15 & 16 \\
\hline 1 & 56 & 92 & 129 & 154 & 180 & 320 & 358 & 396 & 417 & 439 & 443 & 446 & 450 & 454 & 456 & \\
\hline 2 & 47 & 72 & 98 & 123 & 148 & 174 & 195 & 333 & 374 & 413 & 437 & 442 & 446 & 450 & 455 & 456 \\
\hline 3 & 50 & 79 & 107 & 136 & 164 & 193 & 221 & 364 & 392 & 416 & 441 & 446 & 450 & 455 & 456 & \\
\hline 4 & 48 & 75 & 102 & 129 & 163 & 196 & 346 & 382 & 419 & 425 & 431 & 438 & 442 & 447 & 452 & 456 \\
\hline 5 & 55 & 90 & 105 & 160 & 195 & 225 & 349 & 385 & 422 & 429 & 436 & 442 & 448 & 452 & 455 & 456 \\
\hline PV & 49 & 77 & 105 & 133 & 161 & 189 & 333 & 380 & 408 & 434 & 440 & 446 & 450 & 454 & 455 & 456 \\
\hline
\end{tabular}

In practice, EV measurements will be collected as imposed by contract specifications or at distinct time instances. It is easily understood that such time-instances will not always coincide with the discrete percentage complete points for which tolerance limits for control charts are calculated. This is also not the case for the project runs in our simulation software. If data are collected at fixed time-intervals, the measurements along the time-axis need to be transformed towards measurements equally spaced along the percentage complete axis. The last row of table 3 displays the planned value along the project duration. The transformation towards measurements equally spaced along the percentage complete axis is done by means of interpolation.

Interpolation might introduce additional variance in the observations made in both the static phase and the dynamic phase. However, this error-term can be kept within reasonable value if both the increment in percentage complete $\triangle P C$ and the periodic intervals at which data is collected are chosen sufficiently small. We illustrate this procedure with the SPI data obtained for percentage completion value of $p=\{10,20,30,40, \ldots, 90\}$ for the first row of table 2 . The SPI measurements for this fictitious project example are obtained from the data recorded for each day of table 3 by dividing the $\mathrm{EV}$ of the first row by the PV, as is illustrated in table 4 . In order to find the SPI value for this fictitious project execution at e.g. $50 \%$ of the project complete, we need to look for the data we collected that have PC values lying the closest to $50 \%$ : in this case our measured SPI after $t_{A}=5$ and $t_{B}=6$ days. To approximate SPI a simple calculation through linear interpolation shows that:

$$
\begin{aligned}
S P I_{p=50 \%} & =S P I_{t_{A}}+\left(50-P C_{t_{A}}\right) \frac{S P I_{t_{B}}-S P I_{t_{A}}}{P C_{t_{B}}-P C_{t_{A}}} \\
& =1.12+(50-39.5) \frac{1.69-1.12}{70.2-39.5} \\
& =1.31
\end{aligned}
$$

These calculations result in the SPI measurements found in table 5. 
Table 4: The SPI along the first fictitious project execution's duration

\begin{tabular}{ccccccccc}
\hline Time & $\mathbf{1}$ & $\mathbf{2}$ & $\mathbf{3}$ & $\mathbf{4}$ & $\mathbf{5}$ & $\mathbf{6}$ & $\mathbf{7}$ & $\mathbf{8}$ \\
\hline EV & 56 & 92 & 129 & 154 & 180 & 320 & 358 & 396 \\
PC & 12.3 & 20.2 & 28.3 & 33.8 & 39.5 & 70.2 & 78.5 & 86.8 \\
PV & 49 & 77 & 105 & 133 & 161 & 189 & 333 & 380 \\
\hline SPI & 1.14 & 1.19 & 1.23 & 1.16 & 1.12 & 1.69 & 1.08 & 1.04 \\
\hline \hline & & & & & & & & \\
\hline Time & $\mathbf{9}$ & $\mathbf{1 0}$ & $\mathbf{1 1}$ & $\mathbf{1 2}$ & $\mathbf{1 3}$ & $\mathbf{1 4}$ & $\mathbf{1 5}$ & $\mathbf{1 6}$ \\
\hline EV & 417 & 439 & 443 & 446 & 450 & 454 & 456 & 456 \\
PC & 91.4 & 96.3 & 97.1 & 97.8 & 98.7 & 99.6 & 100 & 100 \\
PV & 408 & 434 & 440 & 446 & 450 & 454 & 455 & 456 \\
\hline SPI & 1.02 & 1.01 & 1.01 & 1 & 1 & 1 & 1 & 1 \\
\hline \hline
\end{tabular}

Table 5: The SPI for PC intervals after interpolation

\begin{tabular}{cccccccccc}
\hline $\mathbf{P C}$ & $\mathbf{1 0 \%}$ & $\mathbf{2 0 \%}$ & $\mathbf{3 0 \%}$ & $\mathbf{4 0 \%}$ & $\mathbf{5 0 \%}$ & $\mathbf{6 0 \%}$ & $\mathbf{7 0 \%}$ & $\mathbf{8 0 \%}$ & $\mathbf{9 0 \%}$ \\
\hline $\mathbf{1}$ & 1.11 & 1.19 & 1.21 & 1.13 & 1.31 & 1.5 & 1.69 & 1.07 & 1.03 \\
$\mathbf{2}$ & 0.96 & 0.93 & 0.92 & 0.79 & 0.66 & 0.76 & 0.85 & 0.91 & 0.95 \\
$\mathbf{3}$ & 1.02 & 1.03 & 1.02 & 1.02 & 0.67 & 0.77 & 0.87 & 0.96 & 0.96 \\
$\mathbf{4}$ & 0.98 & 0.97 & 0.98 & 1.03 & 1.04 & 1.04 & 1.04 & 1.02 & 1.03 \\
$\mathbf{5}$ & 1.1 & 1.16 & 1.12 & 1.21 & 1.19 & 1.14 & 1.08 & 1.03 & 1.02 \\
\hline$\overline{X_{p}}$ & 1.03 & 1.05 & 1.05 & 1.03 & 0.98 & 1.04 & 1.11 & 1.00 & 1.00 \\
$L T L_{X_{p}}^{10 \%}$ & 0.97 & 0.95 & 0.94 & 0.88 & 0.66 & 0.76 & 0.86 & 0.93 & 0.95 \\
$U T L_{X_{p}}^{10 \%}$ & 1.11 & 1.18 & 1.17 & 1.18 & 1.26 & 1.36 & 1.45 & 1.05 & 1.03 \\
\hline \hline
\end{tabular}




\section{1. $X$ chart}

To calculate the tolerance limits for the $X$ chart, we need to find the first and last $\alpha$ quantiles for $X_{p}$ at all $P=9$ instances. This illustrative example only includes the SPI as a control variable. For more details regarding the calculations of the other earned value metric (SV) and the earned schedule metrics $(\mathrm{SPI}(\mathrm{t})$ and $\mathrm{SV}(\mathrm{t}))$, we refer the reader to the detailed work by Vanhoucke [12] and the original work on earned schedule by Lipke [33].

Figure 3 illustrates the tolerance limits obtained for an $X$ chart for SPI. The central line $(C L)$ is added for illustrative purposes and is the mean SPI along the project $\left(\bar{X}_{p}\right)$, the outer lines represent the upper and lower tolerance limits $\left(L T L_{X_{p}}^{\alpha}, U T L_{X_{p}}^{\alpha}\right.$, for $\left.\alpha=10 \%\right)$ and the dots illustrate how measurements made on a new run (with durations that exceed the predefined $30 \%$ margins) are categorized as not as planned as soon as its value exceeds a tolerance limit.

Illustrative $X$ chart for project control

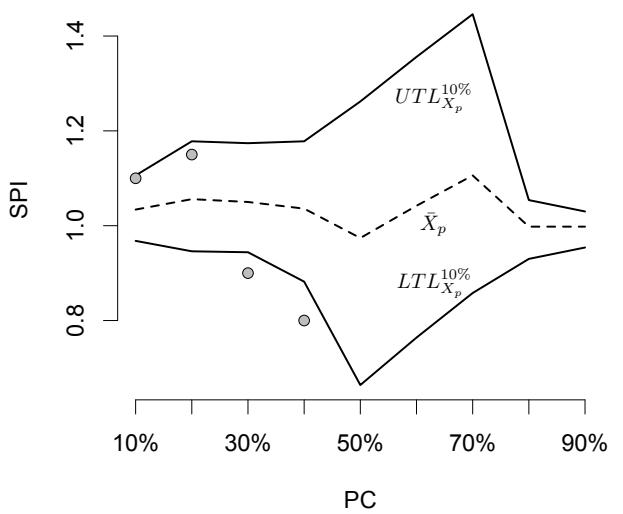

Figure 3: $\mathrm{X}$ chart control limits for the illustrative example

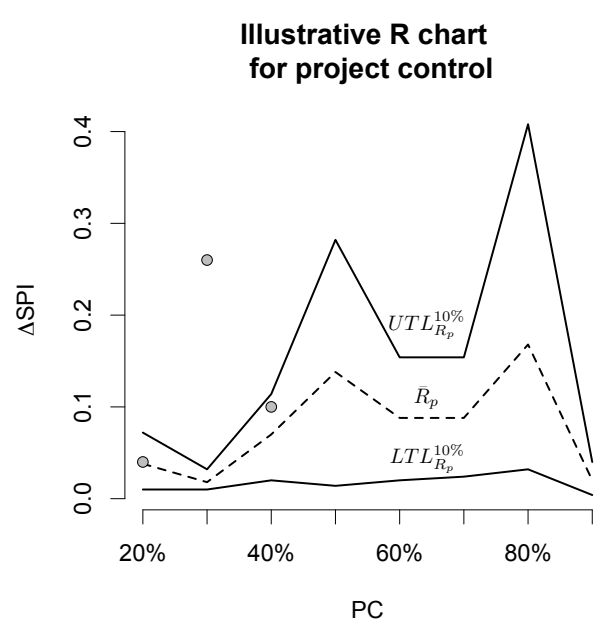

Figure 4: $\mathrm{R}$ chart control limits for the illustrative example

\section{2. $R$ chart}

The $\mathrm{R}$ chart investigates the difference between two consecutive measurements. To illustrate the procedure, the SPI values from table 5 are transformed into the $\Delta$ SPI values of table 6 . Afterwards, the sample mean $\left(\bar{R}_{p}\right)$ and the quantiles are calculated from table 6 . Figure 4 depicts the $\mathrm{R}$ control chart with the same run outlined as figure 3 . This illustrates how both charts can show completely different dynamic behaviour of the calculated control limits, but can be read and processed in entirely the same way.

\section{Research methodology and experimental test design}

In this section, the data generation process to create a project benchmark set, the methodology used, as well as the main research questions set during the computational experiment in order to validate the project control approach are explained along the following subsections. 
Table 6: The $\Delta$ SPI for PC intervals after interpolation

\begin{tabular}{ccccccccc}
\hline $\mathbf{P C}$ & $\mathbf{2 0 \%}$ & $\mathbf{3 0 \%}$ & $\mathbf{4 0 \%}$ & $\mathbf{5 0 \%}$ & $\mathbf{6 0 \%}$ & $\mathbf{7 0 \%}$ & $\mathbf{8 0 \%}$ & $\mathbf{9 0 \%}$ \\
\hline $\mathbf{1}$ & 0.08 & 0.02 & 0.08 & 0.18 & 0.19 & 0.19 & 0.62 & 0.04 \\
$\mathbf{2}$ & 0.03 & 0.01 & 0.13 & 0.13 & 0.1 & 0.09 & 0.06 & 0.04 \\
$\mathbf{3}$ & 0.01 & 0.01 & 0 & 0.35 & 0.1 & 0.1 & 0.09 & 0 \\
$\mathbf{4}$ & 0.01 & 0.01 & 0.05 & 0.01 & 0 & 0 & 0.02 & 0.01 \\
$\mathbf{5}$ & 0.06 & 0.04 & 0.09 & 0.02 & 0.05 & 0.06 & 0.05 & 0.01 \\
\hline$\overline{R_{p}}$ & 0.04 & 0.02 & 0.07 & 0.14 & 0.09 & 0.09 & 0.17 & 0.02 \\
$L T L_{R_{P}}^{10 \%}$ & 0.01 & 0.01 & 0.02 & 0.01 & 0.02 & 0.02 & 0.03 & 0 \\
$U T L_{R_{P}}^{10 \%}$ & 0.07 & 0.03 & 0.11 & 0.28 & 0.15 & 0.15 & 0.41 & 0.04 \\
\hline \hline
\end{tabular}

\subsection{Project data}

The $\mathrm{X}$ and $\mathrm{R}$ project control charts are tested on a set of 900 fictitious projects generated by a project generator RanGen $[34,35]$ that has been used previously in project scheduling and control studies. Vanhoucke [28] uses this dataset to connect network topology information to the optimal control procedure found to monitor the time performance of a project.

The 900 projects are chosen in accordance to the serial/parallel (SP) indicator originally proposed by Vanhoucke et al. [36] and used in previous project control simulation studies [24, 28, 29]. The $\mathrm{SP}$ indicator measures how close a project matches a complete serial or parallel project and is based on the maximal progressive level concept of Elmaghraby [37]. If $m$ is the maximal progressive level, it is defined as the maximum number of activities lying on a single path in the project. If $n$ is the number of activities in the project, it is clear that for a serial project $m=n$. The $S P=(m-1) /(n-1)$ then equals 1 for a serial project. If the largest path in a project consists of only 1 activity, the project network is said to be parallel and $S P=0$. The project dataset consists of 100 projects for 9 intermediate $\mathrm{SP}$ values between 0 and $1(S P=\{0.1,0.2, \ldots, 0.9\})$. If conclusions are formulated regarding a mean value over all 900 projects, it is conjectured that they are general enough to extrapolate over all possible project networks. In the results section 5 , we did not find a significant influence of such serial/parallel network structure on the performance of our control chart to monitor the schedule performance of a project. This result shows that our statistical project control approach provides a more robust solution than regular use of EVM/ES, which was found to improve notably for more serial project networks [28].

\subsection{Methodology}

Monte Carlo simulations are used in the static phase of our statistical project control procedure to construct the tolerance limits used in the $\mathrm{X}$ and $\mathrm{R}$ charts, as well as to emulate real project executions in the dynamic phase where fictitious project progress will be classified using these charts. A distinction is made between both phases in the form of the variation present at the activity level.

In order to generate fictitious project executions in both the static and dynamic phase of the Monte Carlo simulation, we produce activity durations under interactive and compound uncertainties [38]. Therefore, two sources of uncertainty are applied. In section 4.2.1, variation is modelled as probability distributions that are applied to the duration of the project's activities. This variation will result in fictitious project executions that are either as planned or not as planned. In section 4.2 .2 , we discuss the potential dependencies between a project's activities and how project progress can be affected by uncertain events.

For the construction of the tolerance limits we will allow only variation that is acceptable at the activity level. The projects are executed as planned, which represents the desired state of schedule control in the presence of uncertain project-wide events. In the dynamic phase, fictitious project 
progress is generated for which it is possible that the durations of the activities exhibit unacceptable variation. These fictitious project executions are then used to quantify the performance of the proposed control charts in their ability to accurately categorize whether the plan is still adequate or not. Consequently, the project control charts are used to test whether the fictitious project executions are being executed as planned or not as planned.

\subsubsection{Project control input modelling}

Since the occurrence of a project management simulation model in [39], the question has been asked how to accurately represent the uncertainty present in the duration of activities in a real-life project [40]. Since the PERT-beta distributions have been found to fall short, different authors have suggested alternatives. Kuhl et al. [41] proposed the generalized beta distribution [28, 29]. Trietsch et al. [42] theoretically support a claim to use the Parkinson distribution, while the lognormal distribution [43], a mixture of beta and uniform distributions [44] and the doubly truncated normal distribution [45] have also been applied to study stochastic activity networks.

For the purpose of our research we have chosen to use a uniform distribution. However, the presented methodology can be reproduced with any distributional input that is able to accurately represent the stochastic nature of project activity durations. Moreover, we strongly advise a calibration to historical records for the input modelling, if our methodology would be applied in a practical setting. The uniform distribution reflects an absence of interest in the precise distributional characteristics of the project activities by the project manager. In this view on schedule control, the only interest is in the relative deviation of a duration from its baseline estimate. Most of the simulation experiments will incorporate a linear association variable (see section 4.2.2) or have not as planned variation added to them. Therefore, the resulting distribution for $d_{i} / \hat{d}_{i}$, where $\hat{d}_{i}$ represents the baseline estimate for the duration of activity $i$, will most likely not be uniform. Section 5 has a paragraph allocated to the validation of our simulation model using the recently published lognormal core theory [42]. Moreover, a reasonable fit to the proposed generalized beta distribution [41] was also found during the data analysis process. We give more details concerning the parameters used to simulate activity durations in the remainder of this section 4 .

As planned project progress. Variation is added to the baseline duration $\hat{d}_{i}$ of an activity $i$ in the project by a uniform distribution. We define the as planned project schedule executions in the static phase using the lightgrey probability distribution functions presented in figure 5, characterised by a choice for the maximum allowed deviation from the baseline estimate duration $\delta_{a p}$. This distribution is presented relative to the baseline duration. The Monte Carlo samples that are drawn from this distribution therefore need to be multiplied with the baseline duration $\hat{d}_{i}$, in order to model the duration $d_{i}$ of an activity $i$ in a fictitious execution of the project. The maximal relative deviation $\delta_{a p}$ from the baseline estimate duration represents the subjective estimate of the variation on the baseline schedule that is regarded as planned.

Detect both opportunities and dangers. In the dynamic phase, project progress situations are simulated for which the baseline schedule might no longer be adequate. In order to simulate this, variation is added using a not as planned uniform probability distribution that has a standard deviation larger than that of the as planned uniform distribution. Figure 5 shows two illustrative examples of this not as planned uniform distribution, hatched in black, for a low and a high value of $\% A_{n p}$. The variable $\% A_{n p}$ expresses the percentage of the total number of activities $N$ that has a duration that is not confined within the as planned margin $\delta_{a p} . \% A_{n p}$ is therefore proposed to provide an intuitive basis for creating not as planned project progress situations. The larger $\% A_{n p}$, the more the project progress can be regarded as going not as planned and warning signals generated by a project control procedure becomes more desirable. 


\section{Uniform probability distribution functions}
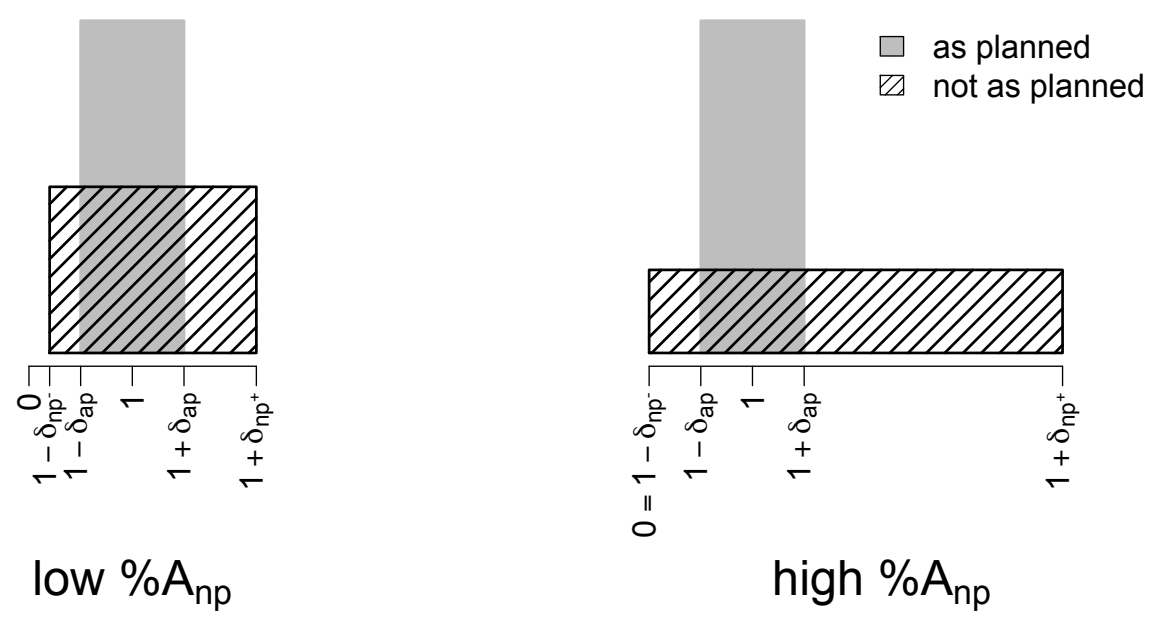

Figure 5: Defining the uniform input model for activity durations

The as planned parameter $\delta_{a p}$ and the not as planned parameter $\% A_{n p}$ will be set in our experiment according to the values specified in table 7 . From the $\delta_{a p}$ value, the extremes of the (lightgrey) as planned uniform probability distribution are calculated directly $\left(1-\delta_{a p}\right.$ and $\left.1+\delta_{a p}\right)$. Indirectly, the extremes of the (hatched in black) not as planned uniform distribution function $\left(1-\delta_{n p^{-}}\right.$ and $\left.1+\delta_{n p^{+}}\right)$can be found using equations 11 and 12 . We will discuss how these extremes are calculated along the following lines.

Let us assume that the $N$ activities in the project have identical probability distributions and that they are sampled independently. The reader should consider the fact that we add dependencies through linear association in section 4.2.2. Let $p_{n p}$ denote the probability of drawing a value outside of the as planned margin. Consider this a success/failure or coin toss experiment where a draw from outside the as planned margin represents a success. The probability of drawing a number of successes $N_{n p}$ larger than $\left\lfloor \% A_{n p} N\right\rfloor$ from a total of $N$ is given by the binomial distribution.

$$
\begin{aligned}
\mathrm{P}\left[N_{n p}>\left\lfloor \% A_{n p} N\right\rfloor\right] & =1-\mathrm{P}\left[N_{n p} \leq\left\lfloor \% A_{n p} N\right\rfloor\right] \\
& =1-\sum_{i=0}^{\left\lfloor \% A_{n p} N\right\rfloor} \frac{N !}{i !(N-i) !} p_{n p}^{i}\left(1-p_{n p}\right)^{N-i}
\end{aligned}
$$

In order to calculate $p_{n p}$ from equation 11 , we have to assign a value to its left-hand side. This value represents the chance that a minimum of $\left\lfloor \% A_{n p} N\right\rfloor$ successes is drawn. We chose this value as $95 \%$ in our experiments, in accordance with a probability of $5 \%$ that a fictitious project with less then $\left\lfloor \% A_{n p} N\right\rfloor$ successes is simulated. For a simulation of 1,000 runs, an average of 50 of such fictitious projects can then be expected to be included in the output. From equation 11, we proceeded with a numerical approximation to calculate $p_{n p}$.

$$
\begin{aligned}
p_{n p} & =\mathrm{P}\left[|D-1|>\delta_{a p}\right] \\
& =1-\frac{2 \delta_{a p}}{\delta_{n p^{+}}+\delta_{n p^{-}}}
\end{aligned}
$$

From $p_{n p}$ and $\delta_{a p}$, the extremes of the not as planned uniform distribution (represented using $\delta_{n p^{-}}$and $\left.\delta_{n p^{+}}\right)$can then be found from equation 12 , where $D$ denotes the random variable that is drawn. 
In order to derive a value for both $\delta_{n p^{-}}$and $\delta_{n p^{+}}$, an assumption has to be made with respect to one or both of these unknowns. We chose to implement a linear relation $\left(\delta_{n p^{+}}=1.5 \delta_{n p^{-}}\right)$ which expresses the tendency of project activities to be late rather than early. However, in the cases where this relation resulted in a negative value for the lower extreme of the distribution $\left(1-\delta_{n p^{-}}<0\right), \delta_{n p^{-}}$was fixed at 1 and the only unknown $\delta_{n p^{+}}$was found from equation 12 . The factor 1.5 shown for the linear relation is arbitrary and different choices were also implemented during our tests.

\subsubsection{Project progress simulation modelling}

The Monte Carlo simulation generates activity durations for all activities according to the input models described in section 4.2.1. In accordance to recent literature we implemented two structures based on the linear association concept [23]. Linear association allows us to model the dependencies between activities and test how project progress is affected by this.

- Independence: The first structure can be regarded as a special case, since complete independence is assumed between activities. Activity durations are sampled independently from each other, but precedence constraints within the project assure that activities influence one another, as expressed in real start times, and the project in its whole. Although this assumption of independence has been shown to fall short in accurately modelling project executions in real life [42, 46, 47], it has been used in both analytical [48, 49] and simulation $[28,29,37,50,51]$ studies to produce insights into stochastic activity networks.

- Linear Association: The second structure models dependencies between activity durations. If users are required to specify correlations between activities, they face a challenging task since there is no established methodology for estimating correlation coefficients. In order to reject the independence assumption in our simulations, we employ the linear association technique [23, 42], where a systemic error $B$ provides objective information on correlations.

We say that $N$ positive random variables $Z_{j}$ are linearly associated if $Z_{j}=B X_{j}$ where $\left\{X_{j}\right\}$ is a set of $n$ independent positive random variables and $B$ is a positive random variable, independent from $\left\{X_{j}\right\}$ that can be regressed from historical data. We follow [23] and model $B$ as a lognormal random variable, since the lognormal distribution is found appropriate to reflect both additive and multiplicative factors, ubiquitous in real-life project activities. The lognormal random variable is characterized by its mean $\mu_{B}$ and its coefficient of variation $c v_{B}$.

Our model assumes that the earned value (EV) for a single activity follows a linear accrue, starting from its actual start up to its budget at completion (BAC) when it is finished [12]. Planned value (PV) follows this same linear accrue from the planned start up to the planned finish time of the activity. EV and PV are calculated in P2 Engine at the project level and are compared to produce $\mathrm{SV}, \mathrm{SPI}, \mathrm{SV}(\mathrm{t})$ and $\mathrm{SPI}(\mathrm{t})$. The calculations for the $\mathrm{X}$ and $\mathrm{R}$ control charts and the recorded performances in section 5 are implemented in the statistical programming language $\mathrm{R}$ [52].

\subsection{Overview of experiments}

Table 7 gives an overview of the simulation experiments conducted for this research. A total of 400 different simulation experiments were run for each project network according to the inputs for the parameters $\mu_{B}, c v_{B}, \delta_{a p}$ and $\% A_{n p}$ provided in table 7 . These parameters define respectively to what extent dependencies between activities are incorporated or not and the as planned and not as planned variation. It should be noted that although the independence assumption is retained for some scenarios (denoted as $c v_{B}=0$ ), a constant $B$ is still used in these to alter the mean $\left(\mu_{B} \in\{0.8,1,1.5,2\}\right)$. The 400 experiments produce 1,000 runs for all 900 projects (section 4.1), for which the baseline estimates and costs are assigned according to the first column of table 7. Periodic EVM/ES reports are generated at 19 distinct moments in the project $(p=$ 
Table 7: Overview of the computational experiments

\begin{tabular}{|c|c|c|}
\hline Baseline & Simulation model & Settings \\
\hline Fstimatos & \multirow{3}{*}{ INDEPENDENCE } & not as planned \\
\hline$\forall i \in \mathcal{N}: \hat{d}_{i} \in[8 ; 56]$ & & $\begin{array}{c}n r s=1,000 \\
\mu_{B} \in\{0.8,1,1.5,2\} \\
c v_{B}=0\end{array}$ \\
\hline $\begin{array}{l}\text { Fixed Cost } \\
\text { Uniform }\end{array}$ & & $\begin{array}{cc}\delta_{a p} \in\{20 \%, . ., 80 \%\} & \delta_{a p} \in\{20 \%, . ., 80 \%\} \\
\% A_{n p}=0 & \% A_{n p} \in\{20 \%, . ., 80 \%\}\end{array}$ \\
\hline $\begin{array}{l}\text { Variable Cost } \\
\text { Uniform } \\
{[700 ; 1500]}\end{array}$ & LINEAR ASSOCIATION & $\begin{array}{c}\text { nrs }=1,000 \\
\mu_{B} \in\{0.8,1,1.5,2\} \\
c v_{B} \in\{0.3,0.8,2\} \\
\delta_{a p} \in\{20 \%, . ., 80 \%\} \quad \delta_{a p} \in\{20 \%, . ., 80 \%\} \\
\% A_{n p}=0 \quad \% A_{n p} \in\{20 \%, . ., 80 \%\}\end{array}$ \\
\hline
\end{tabular}

$\{5 \%, 10 \%, 15 \%, 20 \%, \ldots, 90 \%, 95 \%\})$ and interpolation is done as shown in section 3 with a timeincrement chosen sufficiently small. The durations of the activities were expressed in days, whereas the time-increment was in the order of hours.

We will use these experiments to demonstrate the value of statistical project control for project management in section 5 using the control efficiency measures defined in section 4.4. We have tried to incorporate the best practices for project control modelling as provided by the current literature, to produce results that are as generalisable to project management practice as possible. However, we will expand on the limitations of our research, and its results, in the concluding section 6 .

\subsection{Project control efficiency measures}

In order to measure and compare the efficiency of $\mathrm{X}$ and $\mathrm{R}$ statistical project control charts we propose three simulation-based performance assessment measures [53].

The detection performance. The detection performance equals the frequentist approximation that a certain not as planned situation will result in a signal during the dynamic phase, given the as planned data from the static phase. A signal is generated as in section 2.3 .2 if the project performance $Y_{p}$ or $\left|Y_{p}-Y_{p-1}\right|$ is not confined to the tolerance limits of respectively the $\mathrm{X}$ or $\mathrm{R}$ chart at any review period $p$.

The detection performance is calculated as:

$$
\frac{1}{n r s} \sum_{s=1}^{n r s} \mathbb{1}_{s}\left\{\exists p \mid\left(Y_{p}<L T L_{X_{p}}^{\alpha}\right) \vee\left(Y_{p}>U T L_{X_{p}}^{\alpha}\right)\right\}
$$

for the $\mathrm{X}$ chart, where $\mathbb{1}_{s}\{A\}$ is the indicator function of an event $A$ for a simulation run $s$. Otherwise stated, we represent the occurrence of a signal for the $\mathrm{X}$ charts at review period $p$ as the event $\mathcal{P}_{X}$ :

$$
\frac{1}{n r s} \sum_{s=1}^{n r s} \mathbb{1}_{s}\left\{\exists p \mid \mathcal{P}_{X}\right\}
$$

For the $\mathrm{R}$ chart, the detection performance is calculated analogously as :

$$
\frac{1}{n r s} \sum_{s=1}^{n r s} \mathbb{1}_{s}\left\{\exists p \mid \mathcal{P}_{R}\right\}
$$


where:

$$
\mathbb{1}_{s}\left\{\exists p \mid \mathcal{P}_{R}\right\}=\mathbb{1}_{s}\left\{\exists p \mid\left(\left|Y_{p}-Y_{p-1}\right|<L T L_{R_{p}}^{\alpha}\right) \vee\left(\left|Y_{p}-Y_{p-1}\right|>U T L_{R_{p}}^{\alpha}\right)\right\}
$$

The probability of overreactions. The probability of overreactions approximates the probability that a signal arises even though all activities are executed as planned. This is calculated by 3 -fold cross-validation. The runs of the static phase (going as planned) are divided into 3 equal groups and the probability of overreactions is calculated 3 times. Once for each group where the two other groups are used to calculate the tolerance limits. We confirm that deviations between groups never exceeded $1 \%$, and in the remainder we will present the average over all 3 groups. Consequently, the probability of overreactions represents situations where project managers get a false impression that the project is being executed not as planned, which acts as a trigger to detect the reasons for this behavior, while there is no unacceptable variation present at the activity level. These false signals should be avoided since they lead to time-consuming and unnecessary overreactions made by the project manager and his/her team in charge of controlling the project performance. Each time an not as planned indication is reported, the project control chart is said to be overreacting since it generates a signal, while at activity level only acceptable variation is present.

The probability of overreactions for one group can be calculated using $n r s / 3$ runs from the static phase as (14) and (15) for respectively the $\mathrm{X}$ and the $\mathrm{R}$ chart.

Area under the curve. The probability of overreactions and the detection performance can both be used as approximations of the effectiveness of the proposed project control procedures. However, in the results section 5, the need for a more holistic representation of the effectiveness will be expressed. We find this in the concept of the calculated area under the curve (AUC) for a receiver operating characteristic curve (ROC). The ROC representation is widely used in classification testing and machine learning [54] and can be plotted directly from the probability of overreactions and the detection performance.

Riemann integration of the ROC curve provides us with a single unitless measure for the area under the curve that captures the characteristics of both the probability of overreactions and the detection performance at the same time. The AUC should be more than 0.5, which is the area under the no-discrimination line. This is the line where the true positive rate equals the false positive rate, and which can be regarded as the characteristic of a classification action that is purely based on a randomized process such as a coin toss.

\section{Computational tests and results}

In this section, we present our findings from our large computational experiment. First, we introduce how the concept of the tolerance level $\alpha$ can be used to set a desired degree of probability of overreactions and how the detection performance is affected, in section 5.1. Combined, these two output measures allow us to draw a ROC curve and to calculate the AUC.

Section 5.2 provides the validation for our project progress simulation model according to the recently postulated lognormal core theory [23, 42]. The efficiency of the described $\mathrm{X}$ and $\mathrm{R}$ statistical project control charts is explored in section 5.3 and is compared to a more pragmatic model of traditional EVM/ES use and how it can be implemented for different control points throughout the project in section 5.4

\subsection{Close control versus overreactions - Setting a tolerance level}

When the statistical project control charts are constructed, we use a parameter $\alpha$ as the tolerance level (see section 2.3). This represents the choice for the first and last $\alpha^{\text {th }}$ quantile of the empirical 


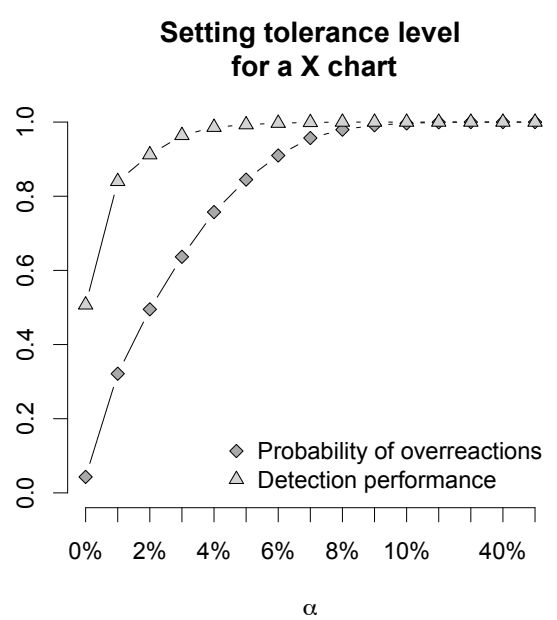

Figure 6: Setting the tolerance level $\alpha$ for a $\mathrm{X}$ chart, for a $\left(\delta_{a p}, \% A_{a p}\right)=(20 \%, 20 \%)$ scenario under the independence assumption $\left(\mu_{B}=1, c v_{B}=0\right)$.
Setting tolerance level

for a R chart

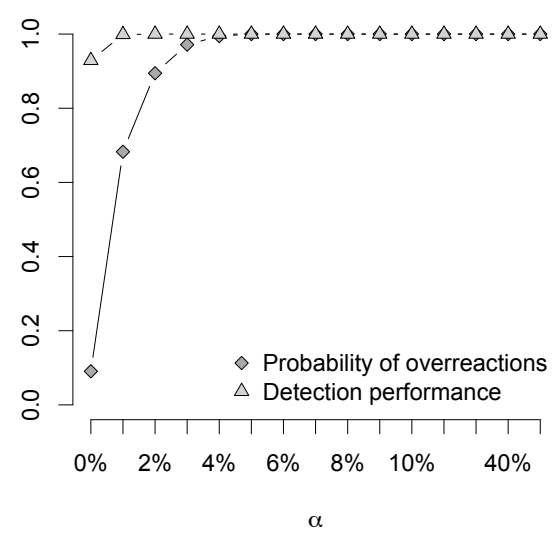

Figure 7: Setting the tolerance level $\alpha$ for a $\mathrm{R}$ chart, for a $\left(\delta_{a p}, \% A_{a p}\right)=(20 \%, 20 \%)$ scenario under the independence assumption $\left(\mu_{B}=1, c v_{B}=0\right)$.

distribution function constructed in the static phase to form the tolerance limits of the $\mathrm{X}$ and $\mathrm{R}$ chart.

Is is clear that $\alpha$ will affect both the probability of overreactions and the detection performance of the $\mathrm{X}$ and $\mathrm{R}$ charts. Therefore, an appropriate choice for $\alpha$ should reflect both a project manager's aversion to risk and his/her willingness to invest effort in false alarms. Figures 6 and 7 illustrate the effect $\alpha$ has on the probability of overreactions and the detection performance for one specific scenario.

It is easily perceived that the real strength of a chart is in the relative improvement of the detection performance over a certain increase in the probability of overreactions. Optimally, we prefer the probability of overreactions as close to 0 as possible. This would mean that the project management team does not need to invest time and effort unnecessarily in drilling down the WBS, to find the variation at the activity level to be confined within the acceptable margins. Analogously, we prefer the detection performance as close to 1 as possible. For a certain scenario, this would imply that the not as planned situation gets detected somewhere throughout the lifetime of the project. This preference is accurately translated into a calculated AUC that needs to be as close to 1 as possible. The ROC curves and the corresponding AUC values for the $\mathrm{X}$ chart and $\mathrm{R}$ chart from figures 6 and 7 are depicted in figure 8 .

\subsection{Validation of the project simulation model}

In order to validate the project progress simulation model (presented in section 4.2.2), we analysed the simulation output according to the lognormal core theory [23, 42]. Figures 9 and 10 depict boxplots for respectively the calculated mean and standard deviations of our simulation output.

Figure 9 presents the mean of the simulated activity durations. This figure clearly shows how the average activity duration in a project is affected by $\mu_{B}$. If $B$ is a random variable $\left(c v_{B} \neq 0\right)$, rather than a constant $\left(c v_{B}=0\right)$, both the spread and the average of the mean duration is increased. Figure 10 shows the effect that the coefficient of variation $c v_{B}$ has on the standard deviation of the simulated activity durations. Both the average and the spread of the standard deviation increase with increasing $c v_{B}$. This effect is noticeably larger for the not as planned situations. 


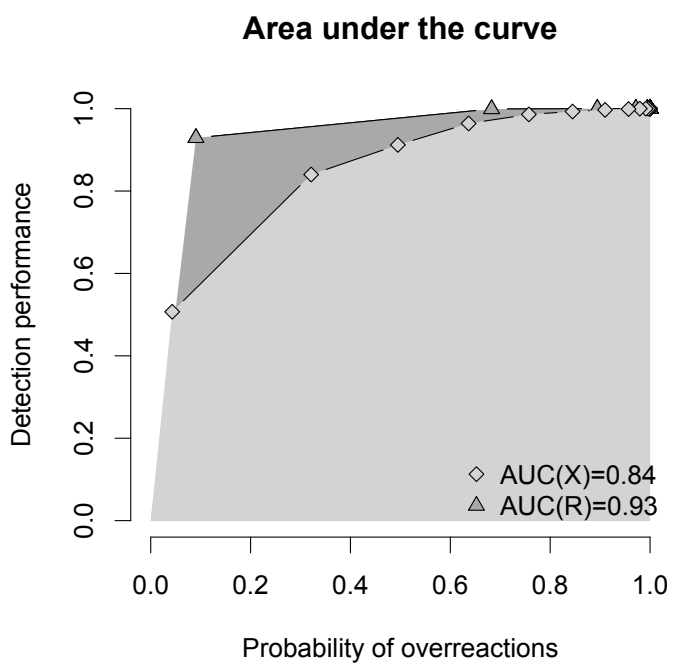

Figure 8: Area under the curve for the X and R chart, for a $\left(\delta_{a p}, \% A_{a p}\right)=(20 \%, 20 \%)$ scenario under the independence assumption $\left(\mu_{B}=1, c v_{B}=0\right)$.

In conclusion, we can safely state that in order to produce project progress simulations with variation in the orders of magnitude that is discussed by Trietsch et al. [42], with high variation instances having a standard deviation larger than 2, and large deviations in the mean, linear association via a lognormal variable $B$ is preferred.

\subsection{Project control efficiency}

This part of the results section explores the efficiency of the proposed control charts using tolerance limits on EVM/ES by means of the area under the curve measure introduced in section 4.4.

\subsubsection{Area under the curve}

The area under the curve representation in figure 11 provides a nuanced and holistic view of the project control efficiency for the $\mathrm{X}$ and the $\mathrm{R}$ chart. In general, the figure shows that the $\mathrm{X}$ and $\mathrm{R}$ chart perform better than that a randomized classification process would. All AUC values are significantly larger than 0.5 , the AUC value of the no-discrimination line. Overall the $\mathrm{X}$ chart outperforms the $\mathrm{R}$ chart. This can be seen more clearly from an aggregated AUC calculated over all simulation experiments. This will be presented in section 5.4

For all cells along the diagonal of the static/dynamic phase grid, the choice for $c v_{B}$ is equal in both phases and we can conclude that the $\mathrm{R}$ chart slightly outperforms the $\mathrm{X}$ chart. In other words, if an historical calibration precedes the simulations of the static phase and new projects can be expected to comply with the historical data, then the tolerance levels of the $\mathrm{R}$ chart prove to be the most efficient project control option. The cells below and above the diagonal represent the performance of the $\mathrm{X}$ and the $\mathrm{R}$ chart under parameter uncertainty [55]. When $c v_{B}$ is expected to underestimate the real coefficient of variation of the linear association variable $B$ during the dynamic phase (above the diagonal in the static/dynamic grid), then the $\mathrm{X}$ chart outperforms the $\mathrm{R}$ chart, although both produce very high AUC values. If however, the coefficient of variation of $B$ is chosen too high (below the diagonal), the $\mathrm{R}$ chart will be more favourable for efficient project control during the dynamic phase. 


\section{Mean activity duration}

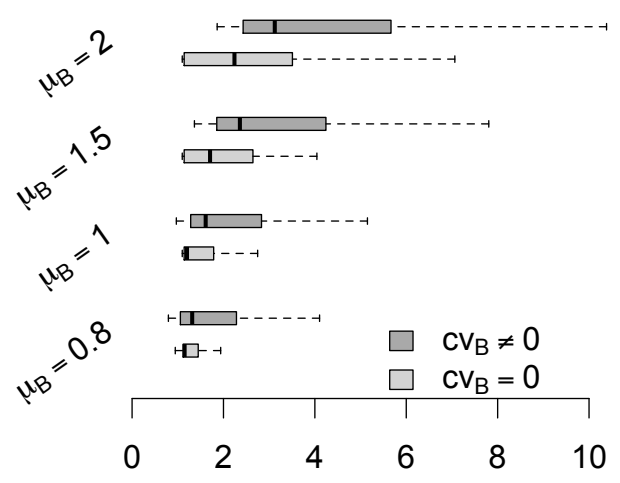

$\mathrm{m}$

Figure 9: Distribution of the resulting mean in the project simulation model.

\section{Standard deviation activity duration}

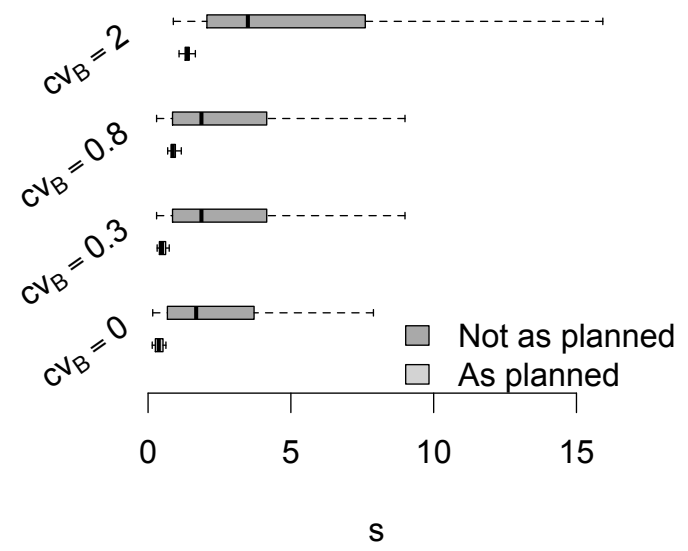

Figure 10: Distribution of the resulting standard deviations in the project simulation model.

\subsubsection{Influencing factors for the $X$ and $R$ chart $A U C$}

We proceed with a discussion in more detail for the AUC shown in figure 11. First, we provide an intuitive basis for the observed variation within each cell of the static/dynamic phase grid. Secondly, we investigate the effect that a change in the mean or coefficient of variation of the observed distribution of $d_{i} / \hat{d}_{i}$ has on the AUC.

\section{Intuitive scenario representation.}

Without loss of generality, we will illustrate the intuitive basis on a single cell of the static/dynamic grid. We assume that the project management team has made the right choice for $c v_{B}=0.3$ and $\mu_{B}=1$ in the static phase from calibration to historical data recorded within the organisation. Moreover, we also assume that the real-life execution, for which the statistical project control charts are calculated, can be correctly modelled using the same random variable $B$. Figure 12 shows the expected AUC over a subset of not as planned scenarios.

In order to improve the readability of figure 12, we applied a color coding where the lightest shades of grey correspond to the lowest values and the darker to the highest values. The depicted scenarios correspond to as planned situations for $\delta_{a p} \in\{20 \%, 40 \%, 60 \%, 80 \%\}$ and not as planned scenarios with $\% A_{n p} \in\{20 \%, 40 \%, 60 \%, 80 \%\}$.

The color-coded variation in figure 12 was found to be identical for both the $\mathrm{X}$ and the $\mathrm{R}$ chart, so only that of the $\mathrm{X}$ chart is shown. Figure 12 largely confirms the intuition behind the scenario representation. The higher the percentage of activities $\% A_{n p}$ that is likely to have a duration that is not confined to the as planned margin $\delta_{a p}$, the more likely this scenario will produce a clear signal that can be interpreted using the statistical project control charts. For any $\% A_{n p} \geq 20 \%$, an increase in AUC can also be observed for increasing values of the as planned margin $\delta_{a p}$. From equation 12 , it can be seen that if $p_{n p}$ remains fixed (resulting from a fixed $\% A_{n p}$ ), then $\left(\delta_{n p^{-}}+\delta_{n p^{+}}\right)$needs to increase if $\delta_{a p}$ increases. The extremes of the not as planned uniform thereby diverge, which results in a larger standard deviation and mean of the distribution. In the next section, it will be shown that increasing values for the mean and standard deviation lead to increasing AUC values. 


\section{Area under the curve}

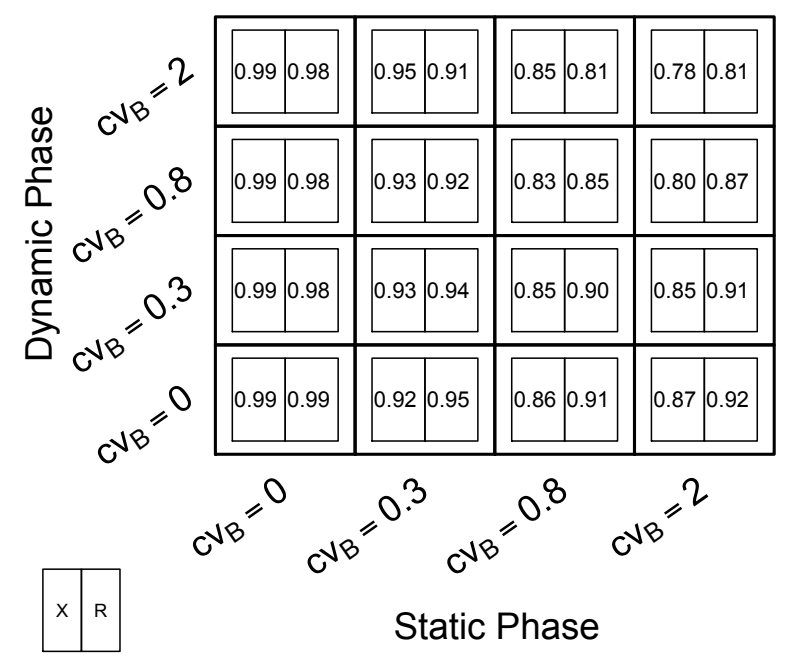

Figure 11: Area under the curve for different static/dynamic phase combinations

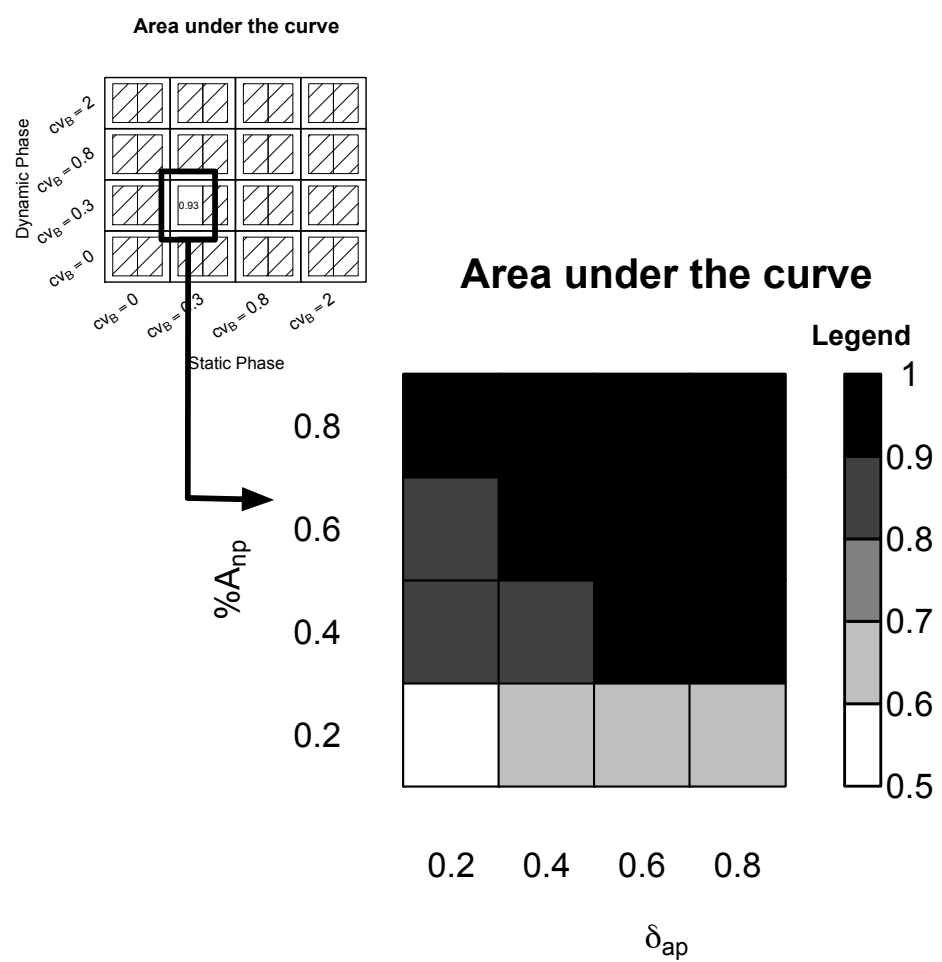

Figure 12: Area under the curve for different not as planned situations 


\section{Influence of a change in mean}

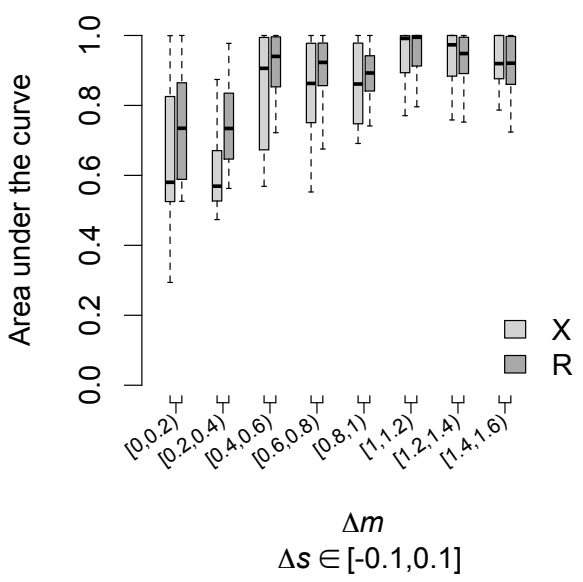

Figure 13: The effect a change in the mean has on the AUC.

\section{Influence of a change in standard deviation}

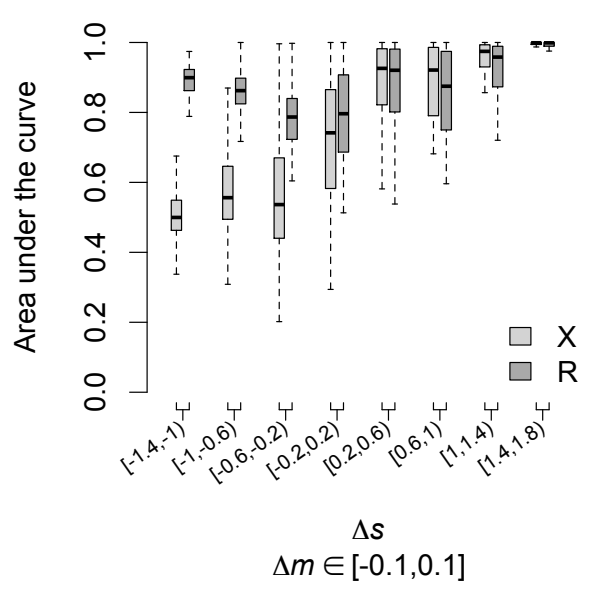

Figure 14: The effect a change in the standard deviation has on the AUC.

Change in mean or standard deviation.

This paragraph explores the effect the observed underlying distribution of $d_{i} / \hat{d}_{i}$ has on the area under the curve. We investigate the impact of a change in mean and standard deviation of the observed distribution of $d_{i} / \hat{d}_{i}$ for the dynamic phase relative to the static phase (which is used to calculate the statistical tolerance limits) for the $\mathrm{X}$ and $\mathrm{R}$ control charts. The relative change in mean $\Delta m=m_{\text {dynamic }}-m_{\text {static }}$ is the difference between the calculated mean of the activity durations in the dynamic phase $\left(m_{\text {dynamic }}\right)$ and the mean of the activity durations in the static phase $\left(m_{\text {static }}\right)$. Similarly, $\Delta s=s_{\text {dynamic }}-s_{\text {static }}$ is defined as the difference between the standard deviation of these two phases.

Figure 13 presents the effect a relative change in the mean of the underlying distribution has on the efficiency of the project control process. The x-axis represents the different ranges of values that are found for $\Delta m$, while the change in the standard deviation is kept close to zero between the dynamic phase and the static phase $(\Delta s \leq \pm 0.1)$. In figure 13, we observe an increasing trend for the AUC for increasing values of $\Delta m$ up to 1.2. If the mean of the static phase is e.g. 1, then increasing values for the AUC can be expected for a mean in the dynamic phase up to 2.2. Both the $\mathrm{X}$ and the $\mathrm{R}$ chart exhibit this behaviour, while the $\mathrm{R}$ chart significantly outperforms the $\mathrm{X}$ chart.

The effect of a change in the standard deviation $\Delta s$ of the underlying distribution is shown in figure 14 for approximately equal mean values $(\Delta m \leq \pm 0.1)$. The $\mathrm{x}$-axis represents the ranges of values found for $\Delta s$. If the standard deviation of the underlying distribution of $d_{i} / \hat{d}_{i}$ decreases between the static and the dynamic phase $(\Delta s<0)$, we observe decreasing values for the AUC of the $\mathrm{X}$ control chart and increasing values for the AUC of the $\mathrm{R}$ control chart. Moreover, the $\mathrm{R}$ chart is preferred in order to accurately detect not as planned situations if $\Delta s<0$. For positive $\Delta s$ values, the $\mathrm{AUC}$ of both the $\mathrm{X}$ chart and the $\mathrm{R}$ chart increases. In these situations, the $\mathrm{X}$ chart performs better than the $\mathrm{R}$ chart.

\subsection{Comparing statistical project control to traditional EVM/ES}

In this section we revisit the discussion on the comparison of the use of project control charts using statistical tolerance limits (STL) to the more traditional use of EVM/ES metrics and some recent advances from literature for project schedule control. Although practical use of EVM/ES 
is typically characterized by on the spot decision-making from practical experience, we implement four techniques to compare our statistical control charts with.

- Rules-of-thumb (RoT): This project schedule control approach combines the use of project control charts with rules-of-thumb. Instead of our statical tolerance limits for the $\mathrm{X}$ chart, these charts employ either static, widening or narrowing tolerance limits for schedule control purposes. The tolerance limits, represented by $a+b p$, are implemented as symmetrical lines around a percentage complete axis. Therefore, the median of each performance metric $\hat{Q}(0.5)_{p}$ calculated in the static phase, is subtracted for all percentage project complete $p$.

- Static tolerance limits mimic the "Target Performance Chart" [56]. The slope a equals 0 , for these tolerance limits

- Widening tolerance limits should be used in combination with SV and SV(t). These variance-based performance metrics grow larger in absolute figures during project progress and therefore, an increasing upper tolerance limit should be used. Since the lower tolerance limit is symmetrical, overall the tolerance limits can be said to be widening towards the end of the project.

- Narrowing tolerance limits should be used in combination with SPI and SPI(t). Simple simulations indicate that the variation for these index-based performance metrics decreases along the percentage complete axis. Consequently, narrowing tolerance limits are preferred during project progress.

Without loss of generality, we assume that these control strategies represent a best-case decision making process from practical experience, since they are calibrated to the static phase simulation data, much as the $\mathrm{X}$ and $\mathrm{R}$ control charts. This calibration is done for the slope $a$ and the intercept $b$, which are optimized using a coarse search algorithm over a large solution space, in order to result in probabilities of overreactions that are competitive with those chosen for the $\mathrm{X}$ and $\mathrm{R}$ chart. In practical decision-making from experience, this calibration is impossible and the real-life probabilities of overreactions can be expected to be much higher. We therefore consider this RoT control strategy to be best-case EVM/ES decision making without the use of statistical tolerance limits.

- EVM/ES on multiple control points: The use of EVM/ES control metrics applied at different (and multiple) control points in the project schedule has recently been discussed by Colin and Vanhoucke [57]. Inspired by the concepts of the critical chain/buffer management (CC/BM) methodology, different control points throughout the project are suggested at which EVM/ES control charts should be monitored. The two alternative approaches are discussed in the original paper and can be briefly summarized as follows

- Feeding paths (FP): The concept of feeding paths is adopted from the CC/BM literature. EVM/ES schedule control metrics are calculated for those paths in the project network where a buffer should be place according to $\mathrm{CC} / \mathrm{BM}$ in order to mitigate risks and to protect the critical path. The schedule control metrics are then monitored on a control chart against rules-of-thumb tolerance levels.

- Sub-networks (SN): The concept of sub-network control points expands on the concept of feeding paths. Schedule control metrics are calculated for the collection of paths that enter the critical path at a given point. Again, these EVM/ES metrics are referenced against tolerance limits on control charts. The tolerance limits are calculated using sample quantiles, in a manner similar to that presented in section 2.3.

It should be noted that the differnece between the two techniques mainly lies in the number of control points, and figure 15 only gives a summary overview of the main results to compare the alternative project control methods. 


\section{Project schedule control using EVM/ES}

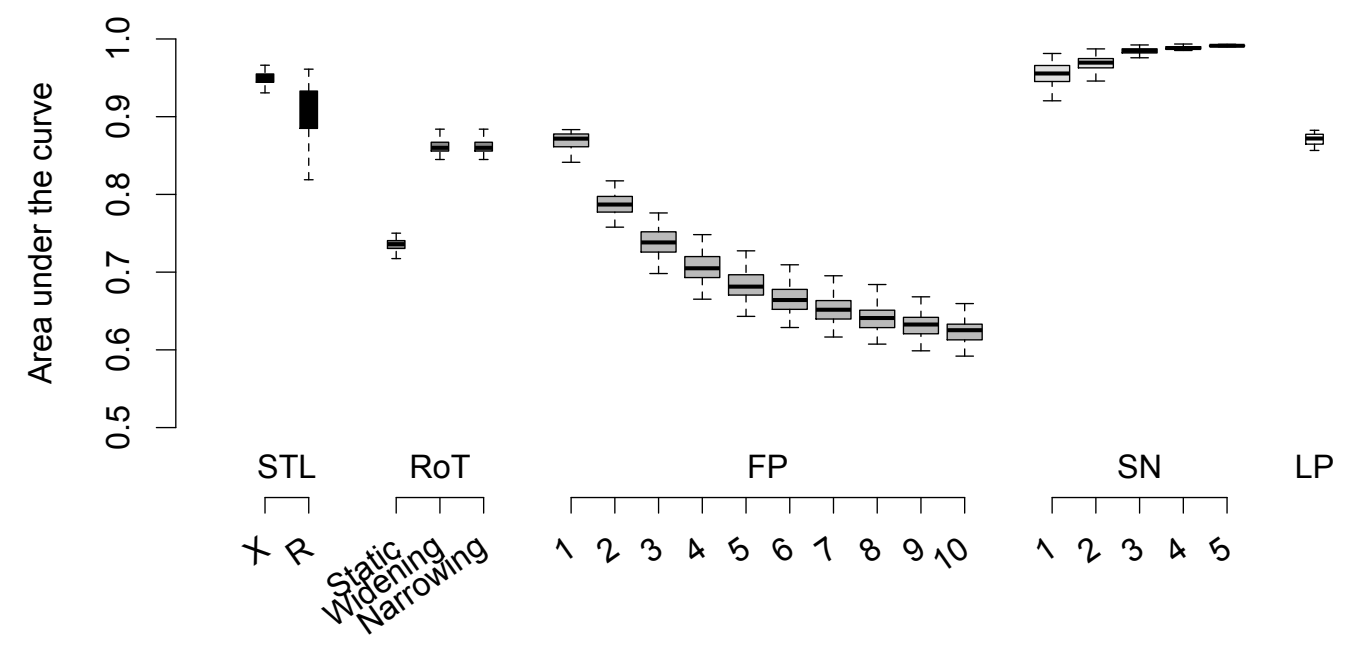

Figure 15: Area under the curve comparison of the X and R charts (STL) with other schedule control approaches using EVM/ES

- Longest path monitoring (LP): Inspired by the fact that the EVM/ES schedule control metrics are fully reliable for serial networks but have a decreasing performance for more parallel networks [28], the longest path control approach was proposed by Lipke [58]. In this method, SPI(t) is referenced against a static tolerance limit on a single project control chart. The $\mathrm{SPI}(\mathrm{t})$ is however not calculated for all activities of the project, but only for those that lie on the longest path in the project network. This longest path is updated dynamically during project progress with actual durations for those activities that have already been finished and the baseline estimate durations for the activities that are not yet started.

Figure 15 depicts the calculated area under the curve as boxplots for the statistical project control charts proposed in this paper and the alternative schedule control procedures outlined in this section. This comparison is based on all the data obtained from the simulations in the static/dynamic phase grid of figure 11. A detailed discussion on the merits and the distinct characteristics of the procedures presented in this section falls outside of the scope of this paper. In short, we conclude that the X chart outperforms the R chart, based on the aggregated AUC over all performed simulation experiments. Both these charts, using statistical tolerance limits should be preferred over all other tested schedule control procedures for the following four reasons

- The RoT strategies display a significantly lower AUC. The narrowing tolerance limits and the widening tolerance limits can however be considered as useful alternatives.

- The FP method is shown for a combination of maximally 10 feeding paths monitored during project progress. The maximal number of feeding paths can however be much larger, even for small projects. The $\mathrm{x}$-axis in figure 15 under FP depicts the number of control points that are used. The first control point is always the critical path, while the others are used to monitor the performance of the feeding paths. When not all feeding paths are monitored, information about certain activities is lost. This results in an increased probability of overreactions, which affects the detection performance and consequently the calculated AUC. Figure 15 shows that this produces a decreasing schedule control performance for an increasing number of included feeding paths.

- The SN method shows the opposite behaviour. AUC increases to the optimal unity area under the curve for 5 sub-network control points included in the schedule control procedure. 
When its performance is compared to the $\mathrm{X}$ chart, it shows to be a valuable alternative. However, while the $\mathrm{X}$ control chart relies on only one control point, the SN approach needs up to 5 control points to only slightly outperform the X chart. Moreover, the SN approach also relies on the STL procedure which is the topic of this paper.

- The LP method [58] is found to be outperformed by both the $\mathrm{X}$ and the $\mathrm{R}$ chart. Using our definition and measures for schedule control it is not able to produce similar results. However, this method was developed foremost to produce project duration forecasts, rather than to detect not as planned project progress.

\section{Discussion and conclusions}

In this paper, a project control system is presented which is inspired by the well-known statistical process control charts widely used to monitor manufacturing processes but is fundamentally different in its use of the progress data to construct tolerance limits. The use of earned value management performance metrics in a control chart, based on simulated random variation, allows to discriminate between as planned and not as planned project performance variation. This paper measures the performance of the two proposed charts, using a wide range of fundamentally different project networks in a large computational experiment. The first chart (X chart) mimics a Shewhart chart for process control with adaptations to fit the finite nature of a project. The $\mathrm{X}$ chart uses the commonly known schedule performance metrics provided in an earned value management system. The second chart ( $\mathrm{R}$ chart) proposes an adjustment on these metrics to focus on the instantaneous changes in project performance. The assumption is that by performing differential calculations, the accumulated performance of past activities is filtered and only active tasks are being monitored.

Overall the $\mathrm{X}$ chart shows to be the most promising to be used in practice to monitor deviations from the baseline schedule. Altogether, both show very comparable performance. The results of various computational experiments show the relevance and usefulness of this approach in a project control setting, although all the results are subject to the specific definition of as planned and not as planned project progress and the characteristics of the simulation model as applied in this research. In order to assess the performance of the statistical tolerance limit approach discussed in the paper, the $\mathrm{X}$ and $\mathrm{R}$ chart's performances are compared to add-hoc rules-ofthumb strategies that would typically be used in project control using EVM/ES and some recently developed EVM/ES extensions. Overall, the X and R chart can defensibly be preferred over all tested procedures

Obviously, the results obtained in this study should be put in the right perspective. The use of simulated data often shows some weaknesses and the results should therefore be interpreted with care. A first assumption in our simulation study lies in the dependence structure of activity durations during sampling. While the assumption of independence was invalidated in previous research $[42,59]$, it has nevertheless been used in earlier reports on project control [28, 29]. We also expanded our project progress simulation model with the concept of linear association [23]. However, the input for our simulation experiments depends largely on subjective estimates for activity durations, where in practice calibration to historical data should always be preferred.

A second restriction of our simulated project control study is the strict focus on time performance of projects. Although EVM/ES takes both a time and cost focus during project performance management, this paper puts a strong and restricted focus on the time dimension of a project in progress in order to validate the use of schedule performance metrics in a statistical project control approach. Activity costs have been assumed to be linearly dependent on the activity duration time, which is restricted to the use of periodic costs. In the future, our model could be adjusted to incorporate other situations. Furthermore, other relevant project performance dimensions, such as quality control or project scope control could not be taken into account in this study. Future 
research will build on this paper, stretching its scope to incorporate the restrictions and expanding on the idea of using process control procedures for project control.

Finally, it should be mentioned that a detailed comparison between the statistical project control approach of this paper and the alternative control approaches available in literature is not within the scope of this paper. A comparison between the quality of methods such as Critical Path Method (CPM, [1]), the Programme Evaluation and Review Technique (PERT, [60]), its novel extension to PERT21 [23], the bottom-up approach using Schedule Risk Analysis [29] and the Critical Chain Project Management (CCPM, [61, 62]) is therefore considered as an interesting future research avenue to further improve and optimize the knowledge on project control.

This study should be relevant for both practitioners and academics for the following reasons. Performance indicators from earned value management/earned schedule have been used widely by practitioners in controlling projects. However, the lack of guidelines towards reasonable tolerance limits to discriminate between acceptable and unacceptable performance variation has often classified this technique as secondary to the necessary skills of intuition, existing experience and knowledge a project manager must have. The methodology and control approach proposed in this paper quantifies the use of tolerance limits. This paper shows that the discriminative power between as planned and not as planned variation in a project is much better when a customized control chart is used rather than when manual and intuitive thresholds (represented through the rules-of-thumb) are used. The on the spot decision-making during project control is thereby assisted. Next to the practical relevance, we also believe that this topic will contribute to new research challenges where extensions of this approach might lead to an increased discriminative power and better project control.

\section{References}

[1] J. Kelley, M. Walker, Critical path planning and scheduling: An introduction, Mauchly Associates, Ambler, PA, 1959.

[2] J. Kelley, Critical path planning and scheduling: Mathematical basis, Operations Research 9 (1961) 296-320.

[3] D. Malcolm, J. Roseboom, C. Clark, W. Fazar, Application of a technique for a research and development program evaluation, Operations Research (1959) 646-669.

[4] S. Hartmann, D. Briskorn, A survey of variants and extensions of the resource-constrained project scheduling problem, European Journal of Operational Research 207 (2010) 1-15.

[5] M. Madadi, H. Iranmanesh, A management oriented approach to reduce a project duration and its risk (variability), European Journal of Operational Research 219 (3) (2012) 751-761.

[6] E. Uyttewaal, Dynamic Scheduling With Microsoft Office Project 2003: The book by and for professionals, Co-published with International Institute for Learning, Inc., 2005.

[7] M. Vanhoucke, Project Management with Dynamic Scheduling: Baseline Scheduling, Risk Analysis and Project Control, Vol. XVIII, Springer, 2012.

[8] S. Rozenes, G. Vitner, S. Spraggett, Project control: literature review, Project Management Journal 37 (2006) 5-14.

[9] S. Rozenes, G. Vitner, S. Spraggett, MPCS: Multidimensional project control system, International Journal of Project Management 22 (2004) 109-118.

[10] Q. Fleming, J. Koppelman, Earned value project management. 3rd Edition, Newtown Square, PA: Project Management Institute, 2005. 
[11] W. Lipke, O. Zwikael, K. Henderson, F. Anbari, Prediction of project outcome: The application of statistical methods to earned value management and earned schedule performance indexes, International Journal of Project Management 27 (2009) 400-407.

[12] M. Vanhoucke, Measuring Time - Improving Project Performance using Earned Value Management, Vol. 136 of International Series in Operations Research and Management Science, Springer, 2010.

[13] D. Hulett, Schedule risk analysis simplified, Project Management Network 10 (1996) 23-30.

[14] Y. Fang, J. Zhang, Performance of control charts for autoregressive conditional heteroscedastic processes, Journal of Applied Statistics 26 (6) (1999) 701-714.

[15] W. A. Shewhart, Economic control of quality of manufactured product, Vol. 509, ASQ Quality Press, 1931.

[16] W. Lipke, A study of the normality of earned value management indicators, The Measurable News 4 (2002) 1,6,7,12-14,16.

[17] W. Lipke, J. Vaughn, Statistical process control meets earned value, CrossTalk: The Journal of Defense Software Engineering June (2000) 16-20,28-29.

[18] G. T. Bauch, C. A. Chung, A statistical project control tool for engineering managers, Project Management Journal 32 (2001) 37-44.

[19] Q. Wang, N. Jiang, L. Gou, M. Che, R. Zhang, Practical experiences of cost/schedule measure through earned value management and statistical process control, Lecture Notes in Computer Science 3966 (2006) 348-354.

[20] S. S. Leu, Y. C. Lin, Project performance evaluation based on statistical process control techniques, Journal of Construction Engineering and Management 134 (2008) 813-819.

[21] R. Aliverdi, L. Moslemi Naeni, A. Salehipour, Monitoring project duration and cost in a construction project by applying statistical quality control charts, International Journal of Project Management.

[22] E. Page, Continuous inspection schemes, Biometrika (1954) 100-115.

[23] D. Trietsch, K. R. Baker, Pert 21: Fitting PERT/CPM for use in the 21st century, International Journal of Project Management 30 (4) (2012) 490-502.

[24] M. Vanhoucke, S. Vandevoorde, A simulation and evaluation of earned value metrics to forecast the project duration, Journal of the Operational Research Society 58 (2007) 1361-1374.

[25] G. Burrows, Statistical tolerance limits-what are they, Tech. rep., Knolls Atomic Power Lab., Schenectady, NY (1962).

[26] R. Van Slyke, Monte Carlo methods and the PERT problem, Operations Research 11 (1963) 839-860.

[27] T. Williams, A classified bibliography of recent research relating to project risk management, European Journal of Operational Research 85 (1995) 18-38.

[28] M. Vanhoucke, Using activity sensitivity and network topology information to monitor project time performance, Omega The International Journal of Management Science 38 (2010) 359370.

[29] M. Vanhoucke, On the dynamic use of project performance and schedule risk information during project tracking, Omega The International Journal of Management Science 39 (2011) $416-426$. 
[30] M. Vanhoucke, Integrated Project Management and Control: First come the theory, then the practice, Management for Professionals, Springer, 2014.

[31] D. C. Montgomery, W. Woodall, Research issues and and ideas in statistical process control, Journal of Quality Technology 31 (4) (1999) 376-387.

[32] R. J. Hyndman, Y. Fan, Sample quantiles in statistical packages, The American Statistician 50 (4) (1996) 361-365.

[33] W. Lipke, Statistical methods applied to EVM ...the next frontier, The Measurable News Winter.

[34] L. Tavares, J. Ferreira, J. Coelho, The risk of delay of a project in terms of the morphology of its network, European Journal of Operational Research 119 (1999) 510-537.

[35] E. Demeulemeester, M. Vanhoucke, W. Herroelen, Rangen: A random network generator for activity-on-the-node networks, Journal of Scheduling 6 (2003) 17-38.

[36] M. Vanhoucke, J. Coelho, D. Debels, B. Maenhout, L. Tavares, An evaluation of the adequacy of project network generators with systematically sampled networks, European Journal of Operational Research 187 (2008) 511-524.

[37] S. Elmaghraby, On criticality and sensitivity in activity networks, European Journal of Operational Research 127 (2000) 220-238.

[38] S. Wang, G. Huang, An integrated approach for water resources decision making under interactive and compound uncertainties, Omega The International Journal of Management Science 44 (2014) 32-40.

[39] T. Williams, What are PERT estimates?, Journal of the Operational Research Society 46 (1995) 1498-1504.

[40] M. E. Kreye, Y. M. Goh, L. B. Newnes, P. Goodwin, Approaches to displaying information to assist decisions under uncertainty, Omega The International Journal of Management Science 40 (6) (2012) 682-692.

[41] M. E. Kuhl, E. K. Lada, N. M. Steiger, M. A. Wagner, J. R. Wilson, Introduction to modeling and generating probabilistic input processes for simulation, in: S. Henderson, B. Biller, M. Hsieh, J. Shortle, J. Tew, R. Barton (Eds.), Proceedings of the 2007 Winter Simulation Conference, New Jersey: Institute of Electrical and Electronics Engineers, 2007, pp. 63-76.

[42] D. Trietsch, L. Mazmanyan, L. Govergyan, K. R. Baker, Modeling activity times by the Parkinson distribution with a lognormal core: Theory and validation, European Journal of Operational Research (2012) 386-396.

[43] S. Mohan, M. Gopalakrishnan, H. Balasubramanian, A. Chandrashekar, A lognormal approximation of activity duration in pert using two time estimates, Journal of the Operational Research Society 58 (6) (2007) 827-831.

[44] E. Hahn, Mixture densities for project management activity times: A robust approach to PERT, European Journal of Operational Research 188 (2008) 450-459.

[45] T. Kotiah, N. D. Wallace, Another look at the pert assumptions, Management Science 20 (1) (1973) 44-49.

[46] C. Ragsdale, The current state of network simulation in project management theory and practice, Omega The International Journal of Management Science 17 (1) (1989) 21-25.

[47] R. Schonberger, Why projects are "always" late: A rationale based on manual simulation of a PERT/CPM network, Interfaces 11 (1981) 65-70. 
[48] D. L. Fisher, D. Saisi, W. M. Goldstein, Stochastic pert networks: Op diagrams, critical paths and the project completion time, Computers \& operations research 12 (5) (1985) 471-482.

[49] B. Dodin, M. Sirvanci, Stochastic networks and the extreme value distribution, Computers \& Operations Research 17 (4) (1990) 397-409.

[50] T. Williams, Practical use of distributions in network analysis, Journal of the Operational Research Society (1992) 265-270.

[51] F. Acebes, J. Pajares, J. M. Galán, A. López-Paredes, A new approach for project control under uncertainty. going back to the basics, International Journal of Project Management.

[52] R Core Team, R: A Language and Environment for Statistical Computing, R Foundation for Statistical Computing, Vienna, Austria (2013).

URL http://www.R-project.org

[53] T. Ponsignon, L. Mönch, Simulation-based performance assessment of master planning approaches in semiconductor manufacturing, Omega The International Journal of Management Science 46 (2014) 21-35.

[54] T. Hastie, R. Tibshirani, J. Friedman, T. Hastie, J. Friedman, R. Tibshirani, The elements of statistical learning, Vol. 2, Springer, 2009.

[55] S. Lim, A joint optimal pricing and order quantity model under parameter uncertainty and its practical implementation, Omega The International Journal of Management Science 41 (6) (2013) 998-1007.

[56] F. Anbari, Earned value project management method and extensions., Project Management Journal 34(4) (2003) 12-23.

[57] J. Colin, M. Vanhoucke, A comparison of the performance of various project control methods using earned value management systems, Submitted to an international journal.

[58] W. Lipke, Speculations on project duration forecasting, The Measurable News 3 (2012) 3-7.

[59] T. Williams, The contribution of mathematical modelling to the practice of project management, IMA Journal of Management Mathematics 14 (1) (2003) 3-30.

[60] W. Fazar, Program evaluation and review technique, The American Statistician 13 (1959) 10.

[61] E. Goldratt, Critical Chain, North River Press, Great Barrington, MA., 1997.

[62] R. C. Ash, P. H. Pittman, Towards holistic project scheduling using critical chain methodology enhanced with pert buffering, International Journal of Project Organisation and Management 1 (2) (2008) 185-203. 